\title{
Noncommutative geometry and nonabelian Berry phase in the wave-packet dynamics of Bloch electrons
}

\author{
Ryuichi Shindou, Ken-Ichiro Imura ${ }^{\dagger \ddagger}$
}

October 27,2018

\begin{abstract}
Motivated by a recent proposal on the possibility of observing a monopole in the band structure, and by an increasing interest on the role of Berry phase in spintronics, we studied the adiabatic motion of a wave packet of Bloch functions, under a perturbation varying slowly and incommensurately to the lattice structure. We show, using only the fundamental principles of quantum mechanics, that the effective wave-packet dynamics is conveniently described by a set of equations of motion (EOM) for a semiclassical particle coupled to a nonabelian gauge field associated with a geometric Berry phase.

Our EOM can be viewed as a generalization of the standard Ehrenfest's theorem, and their derivation was asymptotically exact in the framework of linear response theory. Our analysis is entirely based on the concept of local Bloch bands, a good starting point for describing the adiabatic motion of a wave packet. One of the advantages of our approach is that the various types of gauge fields were classified into two categories by their different physical origin: (i) projection onto specific bands, (ii) timedependent local Bloch basis. Using those gauge fields, we write our EOM in a covariant form, whereas the gauge-invariant field strength stems from the noncommutativity of covariant derivatives along different axes of the reciprocal parameter space. On the other hand, the degeneracy of Bloch bands makes the gauge fields nonabelian.

For the purpose of applying our wave-packet dynamics to the analyses on transport phenomena in the context of Berry phase engineering, we focused on the Hall-type and polarization currents. Our formulation turned out to be useful for investigating and classifying various types of topological current on the same footing. We highlighted their symmetries, in particular, their behavior under time reversal $(T)$ and space inversion $(I)$.
\end{abstract}

\footnotetext{
${ }^{*}$ Department of Physics, University of Tokyo, Hongo 7-3-1, Bunkyo, Tokyo 113-0033, Japan.

${ }^{\dagger}$ Condensed Matter Theory Laboratory, RIKEN (Wako), Hirosawa 2-1, Wako, 351-0198, Japan.

${ }_{\ddagger}$ corresponding author (e-mail: imura@riken.jp).
} 
The result of these analyses was summarized as a set of cancellation rules, schematically shown in Tables 1 and 2. We also introduced the concept of parity polarization current, which may embody the physics of orbital current. Together with charge/spin Hall/polarization currents, this type of orbital current is expected to be a potential probe for detecting and controling Berry phase.

\section{Introduction}

The search for a quantized magnetic monopole has a long history. [1, 2] Recently a group of condensed-matter physicists [3, 4] embodied the idea of detecting a monopole in the band structure. [5] In crystal momentum space, monopoles appear as a source or a sink of the reciprocal magnetic field [6, 7] associated with the geometric phase of Bloch electrons. The geometric phase of a Bloch electron, i.e., its Berry phase, has also attracted much attention on the technological side, in particular, in the context of spintronics. A spin Hall effect has been of much theoretical concern, [8, 9, 10, 11, 12] since it may provide a possible efficient way to induce spin current in a semiconductor sample on which spintronic devices [13] will be constructed.

The subject studied in this paper stands at the interface between the forefront of the search for a monopole and the latest technology of spintronics. We study the wave-packet dynamics of a Bloch electron under pertubations slowly varying in space and in time. We derive and analyze a set of equations of motion (EOM) which describes the center-of-masss motion of such a wave packet together with its internal motion associated with its (pseudo) spin. A reciprocal gauge field of geometric origin (Berry connection) appears naturally in such EOM. 7] Then we combine our formalism with the Boltzmann transport theory to describe such phenomena as spin and orbital transport. Its relevance to quantum charge/spin pumping [14, 15] will be also briefly discussed.

Before going further into the detailed descritption of our project, let us briefly remind you what the Berry phase is, and how it has become to be widely recognized in the community. In his landmark paper [16], Berry introduced it as a quantal phase acquired by a wave function whose Hamiltonian is subject to an adiabatic perturbation. The Berry connection, i.e., a gauge field appears as a phase of the overlap of two wave functions infinitesimally separated in the adiabatic parameter space. Before being formulated in such a systematic manner, the Berry phase, however, had already been recognized and discussed, for somewhat restricted cases though, in several independent contexts. The molecular Aharonov-Bohm effect discussed in Ref. 17 is nothing but a manifestation of Berry phase. Its relevance to band structure had also been recognized in limited situations, such as anomalous Hall effect (AHE), [18, 20 as well as in the study of quantized Hall conductance. [21] The role of Berry phase in ferroelectrics has also been of much theoretical interest. 22] Recently, the Berry phase in AHE has attracted a renewed attention, revealing its rich topological structures. 3, 4, 6, 7, 23, 24, 25, 26, The Berry phase has also been generalized 
to a non-Abelian case. 27 .

The equations of motion (EOM) for a wave packet of Bloch functions ${ }^{1}$ is instrumental in all the analyses done in the paper. In order to illustrate our program, we begin with some details of the description of such EOM. A wave packet of Bloch functions is localized in the phase space around $(\overline{\mathbf{k}}, \overline{\mathbf{x}})$ (where $\overline{\mathbf{k}}$ is a crystal momentum characterizing the Bloch function). The wave packet is also composed of a specific Bloch band $n$, whose energy dispersion relation is given by $\epsilon_{n}^{(0)}(\overline{\mathbf{k}})$. The center of mass coordinates $(\overline{\mathbf{k}}, \overline{\mathbf{x}})$ obey a set of classical EOM, as the Ehrenfest's theorem says. In the presence of electromagnetic field $(\mathbf{E}, \mathbf{B})$, its motion is subject to an electric and Lorentz forces,

$$
\begin{aligned}
\frac{d \overline{\mathbf{k}}}{d t} & =-e\left(\mathbf{E}(\mathbf{x})+\frac{d \overline{\mathbf{x}}}{d t} \times \mathbf{B}(\mathbf{x})\right), \\
\frac{d \overline{\mathbf{x}}}{d t} & =\frac{\partial \epsilon_{n}^{(0)}(\overline{\mathbf{k}})}{\partial \overline{\mathbf{k}}} .
\end{aligned}
$$

These EOM, together with the Boltzmann transport theory, describe the electromagnetic response of the system. To see this point, let us express the charge current in terms of the momentum distribution function $f(\overline{\mathbf{k}})$ as

$$
\mathbf{J}_{\mathcal{C}}=-e \int \frac{d \overline{\mathbf{k}}}{(2 \pi)^{D}} f(\overline{\mathbf{k}}) \frac{d \overline{\mathbf{x}}}{d t}
$$

where $D$ is the dimension of coordinate space. The net current vanishes in the thermal equilibrium. A finite net current appears when either

1. $f(\overline{\mathbf{k}})$ is deviated from its equilibrium value, or

2. $d \overline{\mathbf{x}} / d t$ acquires an anomalous term, i.e., an anomalous velocity.

Case 1 corresponds obviously to the usual ohmic transport, in which the current is induced by a small deformation of a Fermi sphere from its thermally equilibrated distribution. In this case the current is, therefore, carried only by the electrons in the vicinity of the Fermi surface.

The Berry phase contribution to Eq. (3) corresponds to Case 2, and involves, in contrast to Case 1, all the electrons below the Fermi surface. This type of geometric current might be also dissipationless. [10, 28, 29] When Berry connection is taken into account, the classical EOM, in particular, Eq. (2) is subject to a modification. In terms of a reciprocal magnetic field $\mathcal{B}$, the $\mathrm{EOM}$ for $\overline{\mathbf{x}}$ now reads, $[\mathbf{7}$

$$
\frac{d \overline{\mathbf{x}}}{d t}=\frac{\partial \epsilon_{\mathrm{eff}}(\overline{\mathbf{k}}, \overline{\mathbf{x}}, t)}{\partial \overline{\mathbf{k}}}+\frac{d \overline{\mathbf{k}}}{d t} \times \mathcal{B}(\overline{\mathbf{k}}),
$$

where $\epsilon_{\text {eff }}(\overline{\mathbf{k}}, \overline{\mathbf{x}}, t)$ is an effective energy, which will be defined in more precise terms in Eq. (38). The nature of reciprocal magnetic field will be clarified in

\footnotetext{
${ }^{1} \mathrm{~A}$ Bloch function is an eigenstate of a periodic Hamiltonian such as Eq. 10], whose energy spectrum forms a band structure $\epsilon_{n}^{(0)}(\overline{\mathbf{k}})$ defined as in Eq. 11.
} 
Sec. 2. One can observe in Eq. (4) that $\mathcal{B}(\overline{\mathbf{k}})$ acts quite similarly to the Lorentz force in the real space. $\mathcal{B}(\overline{\mathbf{k}})$ encodes information on the topological nature of band structure, in particular, that of band crossings. [7] Indeed, a degeneracy point corresponds to a momopole of $\mathcal{B}(\overline{\mathbf{k}}),[5]$ which has played a crucial role in the understanding of anomalous Hall effect (AHE) 6, 23, 24, 25].

In this paper we study the wave-packet dynamics of Bloch electrons subject to a perturbation $\beta(\mathbf{x}, t)$ varying slowly in space and time. Even though our treatment of $\beta(\mathbf{x}, t)$ is in completely general terms, we can give some concrete examples of $\beta(\mathbf{x}, t)$ as in Ref. [7],

$$
H(\mathbf{p}, \mathbf{x} ; \beta(\mathbf{x}, t))=H_{0}\left(\mathbf{p}+\beta_{\mathbf{1}}(\mathbf{x}, t), \mathbf{x}+\beta_{\mathbf{2}}(\mathbf{x}, t)\right)+\beta_{3}(\mathbf{x}, t) .
$$

$H(\mathbf{p}, \mathbf{x} ; \beta=0)$ is an unperturbed Hamiltonian. The first two categories, $\beta_{\mathbf{1}}(\mathbf{x}, t)$ and $\beta_{\mathbf{2}}(\mathbf{x}, t)$, are in a vectorial form, whereas $\beta_{3}(\mathbf{x}, t)$ is a scalar. In the case of electro-magnetic perturbations, $\beta_{\mathbf{2}}(\mathbf{x}, t)=0$. In the following, we restrict our analyses, for the sake of simplicity, to the case of

$$
\beta_{\mathbf{2}}(\mathbf{x}, t)=0 .
$$

A finite $\beta_{\mathbf{2}}(\mathbf{x}, t)$ could be relevant, e.g., for the study of deformational perturbations in a crystal. [7]

Following the quantum mechanical motion of a wave packet localized around $(\overline{\mathbf{k}}, \overline{\mathbf{x}})$, we study its EOM focusing on the topological nature of band structure, and interpret them in terms of the reciprocal vector potential $\mathcal{A}_{q}$ defined in the $(2 D+1)$-dimensional parameter space $\{q\}=(\overline{\mathbf{k}}, \overline{\mathbf{x}}, t)$. This set of parameters $\{q\}$ plays in our case the role of adiabatic parameters in the original formulation of Berry phase. [16] Our approach is entirely based on the fundamental relations of Schrödinger qauntum mechanics, and makes no referenece to (i) Time-dependent variational principle [7, or (ii) Path-integral method using Wannier basis [30. Although our approach is conceptually much simpler than those mentioned above, this type of analysis can be found, to our knowledge, only in the classical literature. [19, 20, We have in mind a linear response theory with the help of Boltzmann equation. We, therefore, restricted our analysis to the first order of external perturbation $\beta(\mathbf{x}, t)$. We emphasize here that all our analyses are asymptotically exact in the framework of linear response theory.

This paper is organised as follows: In Sec. 2, we first discuss the nature of non-Abelian gauge field, appearing in our EOM, which will be derived later in Sec. 4. In Sec. 3, we state and formulate unambiguously our problem, as well as listing all the assumptions we will make. The EOM is derived in Sec. 4, whose possible application to Berry phase engineering is discussed in Sec. 5, before coming to the conclusions in Sec. 6. Some technical details are left for Appendices.

\section{Origin of the gauge field}

The nature of a reciprocal magnetic field $\mathcal{B}(\overline{\mathbf{k}})$ appeared in Eq. (4) lies, as will be further discussed in Sec. 4, in the noncommutativity of the center of 
mass coordinates, $(\overline{\mathbf{k}}, \overline{\mathbf{x}})$. 10 In more mathematical terms, $\mathcal{B}(\overline{\mathbf{k}})$ is a curvature associated with a geometric Berry connection, i.e., a gauge field. The relation between such noncommutative coordinates as seen in Eqs. (2528) and the MM in momentum space has been of much theoretical interest. 3, 4, 10, 31. From a more general point of view, physics in noncommutative space-time coordinates has been of great theoretical interest, rather in high-energy physics community, in particular, in the context of string and $M$ theories. 32, 33. In the following we consider, instead, the physical origins from which our gauge fields stem, and the mechanism how they are generated, focusing on the case of Bloch electrons under slowly varing perturbation $\beta(\mathbf{x}, t)$.

\subsection{Non-Abelian gauge field, or Berry phase, encoding information on the band structure}

Let us consider the motion of a wave packet composed of a limited number, say, $N$ of degenerate bands over the whole Brillouin zone. When neither the time reversal symmetry nor the spatial inversion symmetry is broken, there always appears a two-fold degeneracy at every k-point (Kramers doublet). If there is no further degeneracy in the system, then $N=2$ in our language. In the following chapters, we will derive, using only the most fundamental relations of Schrödinger quantum mechanics, effective equations of motion (EOM) for this wave packet. These EOM are most conveniently interpreted in terms of nonAbelian gauge fields in the reciprocal space. When we derived these effective equations of motion, we restricted our available Hilbert space to these degenerated bands. In the course of this procedure of projection onto the $N$ bands, all the relevant information, about the bands integrated away, was encoded in the form of a gauge field, and appears in the EOM for the wave packet as a Berry phase.

In order to illustrate this point, let us investigate how those gauge fields are expressed explicitly in terms of Bloch functions. We will see in later sections that the concept of Bloch bands is susceptible of perturbations varying incommensurately to the lattice structure. As a result Bloch electrons become subject to a (non-Abelian) gauge field in a $(2 D+1)$-dimensional parameter space $(\overline{\mathbf{k}}, \overline{\mathbf{x}}, t)$, which we will call below the reciprocal space, from the view point that it is a generalization of the space spanned by $\overline{\mathbf{k}}$, the mean crystal momentum of the wave packet. The reciprocal vector potential takes the form of a $N \times N$ matrix, whose elements are given by

$$
\left(\mathcal{A}_{q}\right)_{m n}=i\left\langle u_{m}(\overline{\mathbf{k}}, \overline{\mathbf{x}}, t) \mid \frac{\partial u_{n}(\overline{\mathbf{k}}, \overline{\mathbf{x}}, t)}{\partial q}\right\rangle
$$

where $q$ should be understood as a general coordinate $q=\bar{k}_{\mu}, \bar{x}_{\nu}, t$ and $\mu, \nu=$ $1, \cdots, D . \quad \bar{k}, \bar{x}$ are center of mass coordinates defined in more precise terms, respectively, in Eqs. (17) and (16). $\left|u_{n}(\mathbf{k}, \overline{\mathbf{x}}, t)\right\rangle=\exp (-i \mathbf{k} \cdot \mathbf{x})\left|\phi_{n}(\mathbf{k}, \overline{\mathbf{x}}, t)\right\rangle$ is the periodic part of a local ${ }^{2}$ Bloch state; $\left\langle\mathbf{x}+\mathbf{a} \mid u_{n}(\mathbf{k}, \overline{\mathbf{x}})\right\rangle=\left\langle\mathbf{x} \mid u_{n}(\mathbf{k}, \overline{\mathbf{x}})\right\rangle$. Inner

${ }^{2}$ The concept of local Bloch function will be briefly introduced in Sec. 2.2 before being 
products involving the periodic part $\left.u_{n}(\mathbf{k}, \overline{\mathbf{x}}, t)\right\rangle$, mean an integration over the unit-cell, with a normalization $\left\langle u_{n}(\mathbf{k}, \overline{\mathbf{x}}, t) \mid u_{n}(\mathbf{k}, \overline{\mathbf{x}}, t)\right\rangle=1$. In the abelian case $N=1$, this vector potential is indeed related to the the reciprocal magnetic field $\mathcal{B}(\overline{\mathbf{k}})$ introduced in Eq. (4) as

$$
\mathcal{B}(\overline{\mathbf{k}})=\frac{\partial}{\partial \overline{\mathbf{k}}} \times \mathcal{A}_{\overline{\mathbf{k}}}
$$

In the non abelian case, the gauge invariant reciprocal field strength should be defined as

$$
\mathcal{F}_{q_{1} q_{2}}=\partial_{q_{1}} \mathcal{A}_{q_{2}}-\partial_{q_{2}} \mathcal{A}_{q_{1}}+i\left[\mathcal{A}_{q_{1}}, \mathcal{A}_{q_{2}}\right]
$$

where $q_{1}, q_{2}=k_{\mu}, \bar{x}_{\mu}, t$. Using a trivial relation $\left\langle\frac{\partial u_{m}}{\partial q} \mid u_{n}\right\rangle+\left\langle u_{m} \mid \frac{\partial u_{n}}{\partial q}\right\rangle=0$, the last term of Eq. (8) can be rewriten as

$$
i \sum_{l=1}^{N}\left(\left\langle\frac{\partial u_{m}}{\partial q_{2}} \mid u_{l}\right\rangle\left\langle u_{l} \mid \frac{\partial u_{n}}{\partial q_{1}}\right\rangle-\left\langle\frac{\partial u_{m}}{\partial q_{1}} \mid u_{l}\right\rangle\left\langle u_{l} \mid \frac{\partial u_{n}}{\partial q_{2}}\right\rangle\right)
$$

whereas,

$$
\left(\partial_{q_{1}} \mathcal{A}_{q_{2}}-\partial_{q_{2}} \mathcal{A}_{q_{1}}\right)_{m n}=i\left(\left\langle\frac{\partial u_{m}}{\partial q_{1}} \mid \frac{\partial u_{n}}{\partial q_{2}}\right\rangle-\left\langle\frac{\partial u_{m}}{\partial q_{2}} \mid \frac{\partial u_{n}}{\partial q_{1}}\right\rangle\right) .
$$

Comparing those two equations, one can immediately see that if $\sum_{l=1}^{N}\left|u_{l}\right\rangle\left\langle u_{l}\right|$ were 1, i.e., if $\left\{\left|u_{l}\right\rangle ; l=1, \cdots, N\right\}$ spanned a complete basis, then $\mathcal{F}_{q_{1} q_{2}}$ would vanish identically. This indicates the fact that the nature of our gauge field lies indeed in the projection of an available Hilbert space onto the relevant $N$ bands. If $\left|u_{l}\right\rangle$ spanned a complete basis, and no band were projected away, there would be no information which should be encoded in the gauge fields. Note also that Eq. (9) takes the familiar form of the Berry curvature in the study of magnetic Bloch bands. 21, 6]

\subsection{Gauge field of two different origins}

The gauge field intorduced in Eq. (7) has two different physical origins :

1. Projection onto a subspace spanned by $N$ Bloch bands,

2. Bloch basis moving in time.

The first point has been already discussed in Sec. 2.1, whereas the second point may need some explanation. In the following sections, we will study the wavepacket dynamics in the phase space in the presence of space and time dependent external pertubation, which varies incommensurately to the lattice structure. In order to define a crystal momentum in such a situation, we replace the spatial coordinate $\mathbf{x}$ in the perturbation $\beta(\mathbf{x}, t)$, introduced as in Eq. (5), by the centerof-mass coordinate $\overline{\mathbf{x}}$ of a wave packet under consideration. This recovers the formulated in more precise terms in Sec. 3 . 
original lattice periodicity of the Hamiltonian, leading us to the concept of local Hamiltonian (Eq. (12) ) and its local Bloch eigenstates (Eq. (13)). The above procedure is justified, whenever the external perturbation varies sufficiently smoothly compared with the width of the wave packet. We then expand the wave packet in terms of the local Bloch eigenstates, $\left|\phi_{n}(\mathbf{k}, \overline{\mathbf{x}}(t), t)\right\rangle$, which evolve as a function of time, both explicitly (through $t$ ) and implicitly (through $\overline{\mathbf{x}}(t))$. This is why our local Bloch function, or rather its periodic part, which has appeared in Eq. (7), depended not only on $\mathbf{k}$ but also $\overline{\mathbf{x}}$ and $t$. Because of the nature of our local Bloch basis, such nontrivial gauge field structure as was introduced in the previous section emerges. To be precise, we had better distinguish between two different types of gauge field (strength) appearing in Eqs. (78): (i) $\mathcal{F}_{\bar{k}_{\mu} \bar{k}_{\nu}}$, (ii) $\mathcal{F}_{\bar{k}_{\mu} \bar{x}_{\nu}}$ and $\mathcal{F}_{\bar{k}_{\mu} t}$. Although the reciprocal field strength introduced in Eq. (8) has various components, i.e., not only (a) $\mathcal{F}_{\bar{k}_{\mu}} \bar{k}_{\nu}, \mathcal{F}_{\bar{k}_{\mu} \bar{x}_{\nu}}$ and $\mathcal{F}_{\bar{k}_{\mu} t}((\mathrm{a})=(\mathrm{i})+(\mathrm{ii}))$, but also (b) $\mathcal{F}_{\bar{x}_{\mu} \bar{x}_{\nu}}$ and $\mathcal{F}_{\bar{x}_{\mu} t}$, the latter components (b) do not appear in our EOM for the wave packet, showing a clear contrast with Ref. [7. However, we will be working in the framework of a linear response theory, and within that framework our EOM turn out to be consistent, when $N=1$, with those of Ref. [7]. This point will be further clarified in Sec. 4.4 by performing a simple power counting analysis.

We will see in detail in Sec. 4 that the two types of gauge field

$$
\begin{aligned}
& \text { 1. } \mathcal{F}_{\bar{k}_{\mu} \bar{k}_{\nu}} \text {, } \\
& \text { 2. } \mathcal{F}_{\bar{k}_{\mu} \bar{x}_{\nu}} \text { and } \mathcal{F}_{\bar{k}_{\mu} t} \text {. }
\end{aligned}
$$

have actually slightly different origins, as well as their different physical consequences which we will discuss in Sec 5 . The former, $\mathcal{F}_{\bar{k}_{\mu}} \bar{k}_{\nu}$, is indeed related to the projection of available Hilbert space onto the relevant degenerate $N$ bands. It yields a finite anomalous velocity, and plays a central role in the understanding of AHE. It appears in the presence of magnetic Bloch bands and ferromagnetic backgrounds. [6, 23, 24, 25]. On the other hand, the latter, $\mathcal{F}_{\bar{k}_{\mu} \bar{x}_{\nu}}$ and $\mathcal{F}_{\bar{k}_{\mu}} t$ appear only in the presence of the time-dependent Bloch basis mentioned above.

\section{Statement of the problem}

Before discussing the EOM in the following section, let us define and formulate our problem here as well as listing all the assumptions we will make. We stress here that all the approximation which we will make are stated here, and that the derivation of the EOM in the following section is indeed exact under the assumptions made in this section.

Let us consider the motion of a wave packet of Bloch functions under perturbations slowly varying in space and time. This perturbation can be, e.g., external electro-magnetic field, as was the case in the study of magnetic Bloch bands. [21, 6] The external perturbation $\beta(\mathbf{x}, t)$, varing incommensurately to the crystal structure, breaks the translational symmetry of the unperturbed 
Hamiltonian,

$$
H_{0}(\mathbf{p}, \mathbf{x})=\frac{\mathbf{p}^{2}}{2 m_{e}}+U(\mathbf{x}), \quad U(\mathbf{x}+\mathbf{a})=U(\mathbf{x}) .
$$

Eigenstates of the above Hamiltonian (10), i.e., Bloch bands (specified by band indices $n$ ) are characterized by crystal momenta $\mathbf{k}$,

$$
H_{0}\left|\phi_{n}^{(0)}(\mathbf{k})\right\rangle=\epsilon_{n}^{(0)}(\mathbf{k})\left|\phi_{n}^{(0)}(\mathbf{k})\right\rangle .
$$

Once the perturbation $\beta(\mathbf{x}, t)$ is switched on, this crystal momentum $\mathbf{k}$ is no longer a good quantum number of the system. However, the typical wave length of the external perturbation is longer by several order of magnitudes than the lattice constants, in a physically relevant parameter regime of our interest. In that case, intermediate length scales do exist, to which our wave packet will belong, in which the external perturbation $\beta(\mathbf{x}, t)$ can be regarded spatially constant at the zero-th order of appoximation. We are thus entitled to consider a wave packet, well localized in this length scale of external perturbation, which has also a peak sharp enough in the space of crystal momentum, moving under pertubations slowly varying in space and time.

Let us now consider a wave packet, $|\Psi(t)\rangle$, localized in the phase space, spanned by the real space coordinate $\mathbf{x}$ and the crystal momentum $\mathbf{k}$, in the vicinity of $(\overline{\mathbf{k}}, \overline{\mathbf{x}})$. For simplicity, and without losing generality, we can assume that the wave packet has a symmetric and smooth shape such that it has a well-distinguished peak at $(\overline{\mathbf{k}}(t), \overline{\mathbf{x}}(t))$ in the phase space, where $\overline{\mathbf{k}}(t)$ and $\overline{\mathbf{x}}(t)$ should coincide with the expectation value of $\mathbf{k}$ and $\mathbf{x}$ at a given time $t$.

Our present goal is to study, as accurately as possible, the quantum mechanical motion of this wave packet, and derive the effective equations of motion for $\overline{\mathbf{x}}$ and $\overline{\mathbf{k}}$. As will soon become clearer, an interpretation in terms of reciprocal gauge field (strength) uncover the nature of various physical phenomena, such as anomalous Hall effect (AHE), 6, 23, 24, 25], spin Hall effect, [10] and quantum charge/spin pumping. 14, 15]

\subsection{Assumption of slowly varying perturbation $\beta(\mathrm{x}, t)$ - concept of the local Hamiltonian and its local Bloch bands}

We consider from now on a perturbation $\beta(\mathbf{x}, t)$ introduced in Eq. (5). As far as the intermediate length scales discussed at the beginning of this section are concerned, $\beta(\mathbf{x}, t)$ can be regarded, over the spread of our wave packet, almost spatially constant. We, therefore, choose, as the starting point of our analysis, a Hamiltonian, dubbed in Ref. [7] as a local Hamiltonian, in which $\mathbf{x}$-dependence of $\beta(\mathbf{x}, t)$ is replaced by $\overline{\mathbf{x}}$, a constant at a given time :

$$
H_{\mathrm{loc}}=H(\mathbf{p}, \mathbf{x} ; \beta(\overline{\mathbf{x}}, t)) .
$$

This $H_{\text {loc }}$ has a very remarkable property ; at a given time $t$ it has the same translational symmetry as the non-perturbed Hamiltonian $H_{0}=H(\mathbf{p}, \mathbf{x} ; \beta=0)$, 
i.e., in other words, $H_{\text {loc }}$ can be diagonalized by a set of local Bloch eigenstates $\left|\phi_{n}(\mathbf{k}, \overline{\mathbf{x}}, t)\right\rangle$ forming a local band $\epsilon_{n}(\mathbf{k}, \overline{\mathbf{x}}, t)$, which now depends on $\overline{\mathbf{x}}(t)$ and $t$ :

$$
H_{\text {loc }}\left|\phi_{n}(\mathbf{k}, \overline{\mathbf{x}}, t)\right\rangle=\epsilon_{n}(\mathbf{k}, \overline{\mathbf{x}}, t)\left|\phi_{n}(\mathbf{k}, \overline{\mathbf{x}}, t)\right\rangle .
$$

We are actually considering a degenerate case where $\epsilon_{n}(\mathbf{k}, \overline{\mathbf{x}}, t)(n=1, \cdots, N)$ takes the same value, which we define to be $\epsilon_{\text {loc }}(\mathbf{k}, \overline{\mathbf{x}}, t)$, i.e.,

$$
\epsilon_{\mathrm{loc}}(\mathbf{k}, \overline{\mathbf{x}}, t) \equiv \epsilon_{1}(\mathbf{k}, \overline{\mathbf{x}}, t)=\cdots=\epsilon_{N}(\mathbf{k}, \overline{\mathbf{x}}, t)
$$

We will see below that the concept of the local Hamiltonian and its assoicated conduction bands plays a central role in the derivation of EOM.

\subsection{Construction of a wave packet}

Superposing local Bloch functions introduced above, we now construct our wave packet. In the spirit of Boltzmann's transport theory, an exchange of energy between the electron and the environment occurs only through scattering events. In the following, we will investigate an adiabatic motion of this wave packet. This picture should be valid over the typical length scale of an adiabatic flight between two scattering events, i.e., over the mean free path of an electron. Let us now proceed step by step, making each logical step as clear as possible.

1. Let us first focus on the real space, in which the electron wave packet is localized around $\overline{\mathbf{x}}$. Then we can compose a wave packet ${ }^{3}$ out of local Bloch functions associated with the local Hamiltonian at $\overline{\mathbf{x}}$ :

$$
|\Psi(t)\rangle=\sum_{n=1}^{N} \int d \mathbf{k} a_{n}(\mathbf{k}, t)\left|\phi_{n}(\mathbf{k}, \overline{\mathbf{x}}, t)\right\rangle .
$$

$a_{n}(\mathbf{k}, t)$ should be normalized properly. The $\overline{\mathbf{x}}$-dependence of $|\Psi(t)\rangle$ is implicit on the left hand side of Eq. (15), which is actually due to the time dependent Bloch basis, $\left|\phi_{n}(\mathbf{k}, \overline{\mathbf{x}}, t)\right\rangle$.

2. In order for the self-consistency, we require that our wave packet (15) does give, the correct expectation value of $\mathbf{x}$, i.e., $\overline{\mathbf{x}}(t)=\left(\bar{x}_{1}(t), \cdots, \bar{x}_{D}(t)\right)$ :

$$
\bar{x}_{\mu}(t)=\left\langle\Psi(t)\left|x_{\mu}\right| \Psi(t)\right\rangle .
$$

This guarantees that our wave packet yield, indeed, the center-of-mass position preassigned in Eq. (12), and that our program makes a selfconsistent closed loop.

Our wave packet (15) can be also regarded as a functional of $a_{n}(\mathbf{k}, t)$, i.e., $|\Psi(t)\rangle=\left|\Psi\left(\left\{a_{n}(\mathbf{k}, t)\right\}\right)\right\rangle$, in which the coefficients $a_{n}(\mathbf{k}, t)$ are chosen so that the

\footnotetext{
${ }^{3}$ As has been discussed at the beginning of this section, the expansion [15] is justified, as far as the spread of wave packet in real space is sufficiently small compared with the typical length scale over which the external perturbation can be regarded almost constant.
} 
self-consistency condition (16) should be satisfied. Eq. (16) is, however, nothing but a weak constraint compared with a huge number of degrees of freedom allowed for $a_{n}(\mathbf{k}, t)$. In order to specify with further precision the coefficients $a_{n}(\mathbf{k}, t)$, we now turn our eyes to the $\mathbf{k}$-space. As has been discussed at the beginning of this section, we can consider, in the length scale of our interest, a wave packet which is localized both in $\mathbf{x}$ and in $\mathbf{k}$. We therefore require, in addition to Eq. (16), that our wave packet should also give the correct expectation value of $k_{\mu}$ :

$$
\bar{k}_{\mu}(t)=\left\langle\Psi(t)\left|k_{\mu}\right| \Psi(t)\right\rangle .
$$

The $\overline{\mathbf{k}}$ dependence of $|\Psi(t)\rangle$ is thus encoded in $a_{n}(\mathbf{k}, t) .{ }^{4}$

In the following section, we will derive the EOM for $\overline{\mathbf{x}}(t)$ and $\overline{\mathbf{k}}(t)$, following the quantum mechanical motion of the wave packet we have just prepared. In order to make the set of EOM self-contained, however, we need also to take care of the motion of internal pseudospin degrees of freedom spanned by $N$ bands. For that purpose it will turn out to be convenient to separate $a_{n}(\mathbf{k}, t)$ into its "phase" or pseudospin part, $\mathbf{z}^{t}(\mathbf{k}, t)=\left(z_{1}(\mathbf{k}, t), \cdots, z_{N}(\mathbf{k}, t)\right)$, and its "amplitude" part, $\rho(k, t)$, by introducing

$$
\begin{aligned}
a_{n}(\mathbf{k}, t) & =\sqrt{\rho(\mathbf{k}, t)} z_{n}(\mathbf{k}, t), \\
\rho(\mathbf{k}, t) & =\sum_{n=1}^{N}\left|a_{n}(\mathbf{k}, t)\right|^{2} .
\end{aligned}
$$

$\left|z_{n}\right|^{2}$ clearly represents the probability that the electron wave packet sits on the $n$-th band among the $N$-fold degenerated bands. Thereby it corresponds to the internal degrees of freedom associated with the wave packet, such as spin and/or orbital, while $\rho(\mathbf{k}, t)$ is the momentum distribution function for the wave packet. Since we assumed that the wave packet is well-localized not only in its real space but also in its reciprocal space, we can assume without any loss of generality the following reduction formula,

$$
\int d \mathbf{k} f(\mathbf{k}, t) \rho(\mathbf{k}, t)=f(\overline{\mathbf{k}}(t), t),
$$

for any sufficiently smooth function $f(\mathbf{k}, t)$. This prescription will be used frequently at the final stage of the derivation of EOM.

\subsection{First order perturbation theory with respect to $\beta(\mathrm{x}, t)$ : a linear response theory}

The wave packet introduced above should obey the Schrödinger equation

$$
i \frac{\partial}{\partial t}|\Psi(t)\rangle=H|\Psi(t)\rangle .
$$

\footnotetext{
${ }^{4}$ So far, our treatment of $\mathbf{x}$ and $\mathbf{k}$ has not been symmetric. This is entirely due to the fact that our perturbation $\beta(\mathbf{x}, t)$ does not depend on $\mathbf{k}$. We can consider, in principle and without much difficulty, such a perturbation that depends on $\mathbf{k}$, and perform symmetric treatment of $\mathbf{x}$ and $\mathbf{k}$. However, in this paper, we restricted ourselves, for the clarity of the paper, to the former case.
} 
As we have briefly seen in the Introduction, our eventual objective is to apply the EOM to the framework of the Boltzmannn transport theory, using formula such as Eq. (3), in order to describe phenomena including the anomalous Hall effect, spin Hall effect and quantum pumping, etc. For that purpose it is enough to consider a linear response of the system, keeping only the terms up to first order of $\partial \beta(\overline{\mathbf{x}}, t) / \partial \overline{\mathbf{x}}$ and $\partial \beta(\overline{\mathbf{x}}, t) / \partial t$.

In the case of the electromagnetic fields, the perturbation $\beta(\overline{\mathbf{x}}, t)$ is embodied by a vector potential $\mathbf{A}(\overline{\mathbf{x}}, t)$, and a scalar potential $A_{0}(\overline{\mathbf{x}})$; the full Hamiltonian reads $H_{0}(\mathbf{x}, \mathbf{p}+e \mathbf{A}(\mathbf{x}, t))-e A_{0}(\mathbf{x})$. Thereby, a linear response to applied electromaganetic fields, $\mathbf{E}=-\partial A_{0} / \partial \mathbf{x}-\partial \mathbf{A} / \partial t, \mathbf{B}=\nabla \times \mathbf{A}$ corresponds to the first order perturbation theory w.r.t. $\beta(\overline{\mathbf{x}}, t)$.

We expand the Hamiltonian in powers of $\mathbf{x}-\overline{\mathbf{x}}$ as

$$
H=H_{\mathrm{loc}}+\frac{1}{2} \sum_{\mu=1}^{D}\left\{\left(x_{\mu}-\bar{x}_{\mu}\right) \frac{\partial H_{\mathrm{loc}}}{\partial \bar{x}_{\mu}}+\frac{\partial H_{\mathrm{loc}}}{\partial \bar{x}_{\mu}}\left(x_{\mu}-\bar{x}_{\mu}\right)\right\},
$$

The first order term on the r.h.s. is written in a symmetrical way in order to keep the Hamiltonian to be hermitian. In the following, based on Eq. (20) we develop a systematic perturbation theory w.r.t. $\beta(\overline{\mathbf{x}}, t)$. In this paper, we focus on the linear response of the system, keeping only the terms upto first order in the expansion. Our treatment is, therefore, self-concistent in the framework of linear response theory.

\section{Equations of motion}

In this section, we sketch the derivation of EOM, paying particular attention to, how the two different types of reciprocal field strength, introduced in Sec. 2, appear in the EOM. Before going into the details of the derivation of EOM, let us remind you that there are two possible sources of Berry curvature in the reciprocal parameter space :

1. projection of available Hilbert space onto the degenerated $N$ Bloch bands,

2. local Bloch basis changing gradually in the course of time.

The time dependence of the local Bloch basis stems, not only from the explicit $t$ dependence of the local Hamiltonian, $H_{\text {loc }}$, but also from our self-consistent treatment of the problem, where the local Hamiltonian depends on the centerof-mass position of the electron wave packet through the external perturbation, $\beta(\overline{\mathbf{x}}(t), t)$.

In many respects, our point of view is reminiscent of the standard Ehrenfest's theorem of quantum mechanics : the expectation value of an operator, such as $\mathbf{x}$ or $\mathbf{p}$, obeys a classical EOM. We actually follow the same type of procedure as the derivation of the Ehrenfest's theorem, and in this sense our EOM can be regarded as a generalized Ehrenfest's theorem for Bloch electrons under perturbations varying slowly in space and time. We will come back to this point later. 


\subsection{Preliminaries}

We investigate, in this section, time evolution of the wave packet constructed in Eq. (15). We are interested, not only in its motion in the phase space, but also in the motion of its internal spin/orbital degrees of freedom. In Sec. 5 we will develop further analyses, from the viewpoint of transport phenomena, on the dynamics associated with such internal degrees of freedom. Having in mind applications of our formalism to those fields, we formulate our equations as generally as possible. More concretely, we consider the time evolution of an arbitary observable, $\mathcal{O}$, or rather of its expectation value,

$$
\overline{\mathcal{O}}(t)=\langle\Psi(t)|\mathcal{O}| \Psi(t)\rangle .
$$

Since we have adopted, for the sake of simplicity, the Schrödinger picture, as seen in Eq. (19), the wave functions evolve in time, while observables are timeindependent. We develop later more detailed analyses on the EOM focusing on the case where $\mathcal{O}=x_{\mu}$ or $k_{\mu}$, but we consider a general observable $\mathcal{O}$ as far as possible in formulating our equations. This will make it easier to apply our formalism to further studies on the dynamics associated with the internal degrees of freedom of Bloch electron.

Having those in mind, let us consider the expectation value of an arbitrary observable $\mathcal{O}$,

$$
\overline{\mathcal{O}}(t)=\sum_{m, n=1}^{N} \int d \mathbf{k} d \mathbf{k}^{\prime} a_{m}^{*}\left(\mathbf{k}^{\prime}, t\right)\langle\mathcal{O}\rangle_{m n}\left(\mathbf{k}^{\prime}, \mathbf{k}\right) a_{n}(\mathbf{k}, t),
$$

where we have introduced an abbreviated notation for the matrix elements of an operator $\mathcal{O}$ evaluated in the restricted subspace spanned by $N$ Bloch bands, i.e.,

$$
\langle\mathcal{O}\rangle_{m n}\left(\mathbf{k}^{\prime}, \mathbf{k}\right)=\left\langle\phi_{m}\left(\mathbf{k}^{\prime}, \overline{\mathbf{x}}, t\right)|\mathcal{O}| \phi_{n}(\mathbf{k}, \overline{\mathbf{x}}, t)\right\rangle .
$$

Note that in this restricted Hilbert space not only $k_{\mu}, x_{\mu}$ or $H$ but also $\frac{\partial}{\partial t}$ are considered to be an operator $\mathcal{O}$. $\langle\mathcal{O}\rangle_{m n}\left(\mathbf{k}^{\prime}, \mathbf{k}\right)$ is generally a $N \times N$ matrix for given $\left(\mathbf{k}^{\prime}, \mathbf{k}\right)$, whereas for a given $(m, n)$, it has, in general, off-diagonal matrix elements, and can be also regarded as a matrix in $\mathbf{k}$-space. The presence of finite off-diagonal matrix elements of $\langle\mathcal{O}\rangle_{m n}\left(\mathbf{k}^{\prime}, \mathbf{k}\right)$, either in the $k$ space or in the pseudospin space prevents some observables from commuting each other, thereby makes them non-abelian.

Let us first consider two concrete examples :

1. Case of $\mathcal{O}=x_{\mu}$ : The matrix elements of an observable $x_{\mu}$ are

$$
\left\langle x_{\mu}\right\rangle_{m n}\left(\mathbf{k}^{\prime}, \mathbf{k}\right)=i \delta\left(\mathbf{k}^{\prime}-\mathbf{k}\right) \delta_{m n} \frac{\partial}{\partial k_{\mu}}+\delta\left(\mathbf{k}^{\prime}-\mathbf{k}\right)\left(\mathcal{A}_{k_{\mu}}\right)_{m n}
$$

The first term is off-diagonal in $\mathbf{k}$-space, when $\mathbf{k}$ is discrete, due to the k-derivative, but is diagonal w.r.t. the band index. In Eq. (23), we kept both $\mathbf{k}^{\prime}$ and $\mathbf{k}$ indices in order to emphasize the fact that this first term 
is off-diagonal. In the following, we will omit quite frequently the $\mathbf{k}^{\prime}$ index, pretending that $\left\langle x_{\mu}\right\rangle_{m n}\left(\mathbf{k}^{\prime}, \mathbf{k}\right)$ is diagonal in $\mathbf{k}$-space after $\delta\left(\mathbf{k}^{\prime}-\mathbf{k}\right)$ is intergrated away. On the contrary, the second term is diagonal in $\mathbf{k}-$ space, but the reciprocal vector potential $\mathcal{A}_{\mathbf{k}}$ defined similarly to Eq. (7), as,

$$
\left(\mathcal{A}_{k_{\mu}}\right)_{m n}=i\left\langle u_{m}(\mathbf{k}, \overline{\mathbf{x}}, t) \mid \frac{\partial u_{n}(\mathbf{k}, \overline{\mathbf{x}}, t)}{\partial k_{\mu}}\right\rangle,
$$

has off-diagonal matrix element between different bands. In the above equations we did not write down the explicit $t$-dependence of $\overline{\mathbf{x}}(t)$ in the brackets.

2. Case of $\mathcal{O}=k_{\mu}$ : This case is even simpler. The crystal momentum $\mathbf{k}$ is diagonal both in $\mathbf{k}$ and in pseudospin indices,

$$
\left\langle k_{\mu}\right\rangle_{m n}\left(\mathbf{k}^{\prime}, \mathbf{k}\right)=\delta\left(\mathbf{k}^{\prime}-\mathbf{k}\right) \delta_{m n} k_{\mu} .
$$

Let us further investigate the off-diagonal components of $\left\langle x_{\mu}\right\rangle_{m n}\left(\mathbf{k}^{\prime}, \mathbf{k}\right)$. We focus here on its commutation relation in $\mathbf{k}$-space. Since the first term on the r.h.s. of Eq. (23) is off-diagonal and the second term is not proproportional to an identity matrix, these two terms do not commute each other. One can indeed verify

$$
\left[\left\langle x_{\mu}\right\rangle,\left\langle x_{\nu}\right\rangle\right]_{m n}(\mathbf{k})=i \mathcal{F}_{k_{\mu} k_{\nu}}
$$

where $[A, B]=A B-B A$ is a standard commutator of two $N \times N$ matrices $A, B$. Thus the noncommutativity of $\left\langle x_{\mu}\right\rangle_{m n}(\mathbf{k})$ turns out to be the origin of the emergence of $\mathcal{F}_{k_{\mu} k_{\nu}}$. Another important remark on the Eq. (23) is that it leads us to introduce naturally the concept of covariant derivative in momentum space 10, defined as

$$
\left(\nabla_{k_{\mu}}\right)_{m n}=\delta_{m n} \frac{\partial}{\partial k_{\mu}}-i\left(\mathcal{A}_{k_{\mu}}\right)_{m n} .
$$

In terms of the covariant derivative $\left(\nabla_{k_{\mu}}\right)_{m n}$ thus introduced, the matrix elements $\left\langle x_{\mu}\right\rangle_{m n}(\mathbf{k})$ can be rewritten simply as

$$
\left\langle x_{\mu}\right\rangle_{m n}(\mathbf{k})=i\left(\nabla_{k_{\mu}}\right)_{m n} .
$$

The commutator between two covariant derivatives along different axes is directly related a non-abelian Berry curvature ;

$$
\left[\nabla_{k_{\mu}}, \nabla_{k_{\nu}}\right]_{m n}=-i \mathcal{F}_{k_{\mu} k_{\nu}} .
$$

In geometric terms, Eq. (28) can be interpreted in such a way that two parallel transports along different axes on a curved surface generally do not commute each other. 


\subsection{To derive the EOM}

Let us now consider the time derivative of the expectation value, $\overline{\mathcal{O}}(t)$. Expanding $|\Psi(t)\rangle$ in terms of the local Bloch functions as Eq. (15), one can classify the time derivative of $\overline{\mathcal{O}}(t)$ into three parts :

$$
\begin{aligned}
\frac{d \overline{\mathcal{O}}(t)}{d t} & =\sum_{\sigma, \sigma^{\prime \prime}} \int d \mathbf{k} d \mathbf{k}^{\prime \prime} \frac{\partial a_{\sigma}^{*}(\mathbf{k}, t)}{\partial t}\langle\mathcal{O}\rangle_{\sigma \sigma^{\prime \prime}}\left(\mathbf{k}, \mathbf{k}^{\prime \prime}\right) a_{\sigma^{\prime \prime}}\left(\mathbf{k}^{\prime \prime}, t\right) \\
& +\sum_{\sigma^{\prime} \sigma^{\prime \prime}} \int d \mathbf{k}^{\prime} d \mathbf{k}^{\prime \prime} a_{\sigma^{\prime}}^{*}\left(\mathbf{k}^{\prime}, t\right)\left(\frac{\partial}{\partial t}\langle\mathcal{O}\rangle_{\sigma^{\prime} \sigma^{\prime \prime}}\left(\mathbf{k}^{\prime}, \mathbf{k}^{\prime \prime}\right)\right) a_{\sigma^{\prime \prime}}\left(\mathbf{k}^{\prime \prime}, t\right) \\
& +\sum_{\sigma^{\prime} \sigma} \int d \mathbf{k}^{\prime} d \mathbf{k} a_{\sigma^{\prime}}^{*}\left(\mathbf{k}^{\prime}, t\right)\langle\mathcal{O}\rangle_{\sigma^{\prime} \sigma}\left(\mathbf{k}^{\prime}, \mathbf{k}\right) \frac{\partial a_{\sigma}(\mathbf{k}, t)}{\partial t}
\end{aligned}
$$

We have in mind that the operator $\mathcal{O}$ is either $x_{\mu}, k_{\mu}$ or some other observables. In the case of standard Ehrenfest's theorem,

$$
\frac{d}{d t}\langle\psi|\mathcal{O}| \psi\rangle=i\langle\psi|[H, \mathcal{O}]| \psi\rangle
$$

the second term of Eq. (29) does not exist, since the matrix element, $\langle\mathcal{O}\rangle_{m n}\left(\mathbf{k}^{\prime}, \mathbf{k}\right)$ is time-dependent only when the local Bloch basis evolves in time. The first and the third terms, i.e., the change of expansion coefficients $a_{\sigma}(\mathbf{k}, t)$ yields a commutator, $[H, \mathcal{O}]$. They contain, however, also a Berry connection contribution, which, together with the second term, produce a new type of contribution, which we will call $\Omega_{\mathcal{O}}^{(2)}$ in Eq. (33). The first term of Eq. (33), $\Omega_{\mathcal{O}}^{(1)}$, is a generalizaton of the standard Ehrenfest's commutator $[H, \mathcal{O}]$, which induces, when $\mathcal{O}=x_{\mu}$, $\mathcal{F}_{\bar{k}_{\mu} \bar{k}_{\nu}}$ in the EOM. On the other hand, the second term, $\Omega_{x_{\mu}}^{(2)}$, can be rewritten in terms of $\mathcal{F}_{\bar{k}_{\mu} \bar{x}_{\nu}}$ and $\mathcal{F}_{\bar{k}_{\mu} t}$.

In order to rewrite the Eq. (29) in terms of $\Omega_{\mathcal{O}}^{(1)}$ and $\Omega_{\mathcal{O}}^{(2)}$, let us first look into the following relation,

$$
\frac{\partial a_{n}^{*}(\mathbf{k}, t)}{\partial t}=\sum_{m=1}^{N} a_{m}^{*}(\mathbf{k}, t)\left[\left\langle\frac{\partial}{\partial t}\right\rangle_{m n}+i\langle H\rangle_{m n}(\mathbf{k})\right] .
$$

The first term is a Berry connection contribution. As is clear when it is written more precisely as

$$
\left\langle\frac{\partial}{\partial t}\right\rangle_{m n}=\left\langle u_{m} \mid \frac{\partial u_{n}}{\partial t}\right\rangle=\left\langle u_{m}(\mathbf{k}, \overline{\mathbf{x}}(t), t)\left|\frac{\partial}{\partial t}\right| u_{n}(\mathbf{k}, \overline{\mathbf{x}}(t), t)\right\rangle,
$$

it emerged as a result of the time evolution of the local Bloch basis. On the other hand, the second term of Eq. (30) yields the commutation $[H, \mathcal{O}]_{m n}$ in Eq. (33). Note also that the derivative $\partial / \partial t$ in Eq. (30) picks up both the explicit and implicit $t$-dependence. Correspondingly, one can also rewrite the first term of Eq. (30) using two types of the gauge field introduced in Sec. 2, i.e.,

$$
\left\langle\frac{\partial}{\partial t}\right\rangle_{m n}=\left(\mathcal{A}_{\bar{x}_{\nu}}\right)_{m n} \frac{d \bar{x}_{\nu}}{d t}+\left(\mathcal{A}_{t}\right)_{m n} .
$$


Our next objective is to calculate the time derivative of an operator such as $\overline{\mathbf{k}}, \overline{\mathbf{x}}$ and express them in such a way that their interpretation in terms of the reciprocal field strength will become as easy as possible. For that purpose, we rearrange the terms in Eq. (29) into two parts, $\Omega_{\mathcal{O}}^{(1)}$ and $\Omega_{\mathcal{O}}^{(2)}$ as, ${ }^{5}$

$$
\begin{aligned}
\frac{d}{d t} \overline{\mathcal{O}}(t) & =\Omega_{\mathcal{O}}^{(1)}+\Omega_{\mathcal{O}}^{(2)} \\
\Omega_{\mathcal{O}}^{(1)} & =i \sum_{m, n=1}^{N} \int d \mathbf{k}_{\mathbf{1}} d \mathbf{k}_{\mathbf{2}} a_{m}^{*}\left(\mathbf{k}_{\mathbf{1}}\right)[\langle H\rangle,\langle\mathcal{O}\rangle]_{m n}\left(\mathbf{k}_{\mathbf{1}}, \mathbf{k}_{\mathbf{2}}\right) a_{n}\left(\mathbf{k}_{\mathbf{2}}\right), \\
\Omega_{\mathcal{O}}^{(2)} & =\sum_{m, n=1}^{N} \int d \mathbf{k}_{\mathbf{1}} d \mathbf{k}_{\mathbf{2}} a_{m}^{*}\left(\mathbf{k}_{\mathbf{1}}\right)\left\{\left[\left\langle\frac{\partial}{\partial t}\right\rangle,\langle\mathcal{O}\rangle\right]_{m n}\left(\mathbf{k}_{\mathbf{1}}, \mathbf{k}_{\mathbf{2}}\right)\right. \\
& \left.+\left(\frac{\partial}{\partial t}\langle\mathcal{O}\rangle_{m n}\left(\mathbf{k}_{\mathbf{1}}, \mathbf{k}_{\mathbf{2}}\right)\right)\right\} a_{n}\left(\mathbf{k}_{\mathbf{2}}\right)
\end{aligned}
$$

In Eq. (33) we did not write down explicitly, for the sake of simplicity, the dependence on $\overline{\mathbf{x}}$ and the $t$ in $\left|\phi_{n}(\mathbf{k})\right\rangle=\left|\phi_{n}(\mathbf{k}, \overline{\mathbf{x}}(t), t)\right\rangle$.

As has been announced in advance, $\Omega_{\mathcal{O}}^{(1)}$ is a generalization (or, rather a restricted version) of the standard Ehrenfest's commutator, coming exclusively from the first and third terms of Eq. (29), whereas $\Omega_{\mathcal{O}}^{(2)}$ is a new type of contribution, which is a collection of Berry curvature terms from all the three parts of Eq. (29). Not only have they different origins, but also are they susceptible of different physical interpretations in terms of the reciprocal field strength. We will see in Sec. 4.2 that in the particular case of $\mathcal{O}=x_{\mu}$, the two contributions, $\Omega_{x_{\mu}}^{(1)}$ and $\Omega_{x_{\mu}}^{(2)}$ are related actually to the two different parts of the gauge field introduced in Sec. 2, i.e., (i) $\mathcal{F}_{k_{\mu} k_{\nu}}$, and (ii) $\mathcal{F}_{k_{\mu} x_{\mu}}, \mathcal{F}_{k_{\mu} t}$.

Having in mind what has been stated above, we can now derive the EOM for $\bar{x}_{\mu}(t)$ and $\bar{k}_{\mu}(t)$. Let us first consider the case of $\mathcal{O}=k_{\mu}$. As seen in Eq. (24), the momentum operator $k_{\mu}$ is not only diagonal in $\mathbf{k}$ coordinates and band indices, but also its matrix element is time-independent. Thus only the first term $\Omega_{k_{\mu}}^{(1)}$ contributes to its EOM . Futhermore, among various matrix elements of the Hamiltonian given in (36 37), only those terms which contain off-diagonal matrix elements w.r.t. $\mathbf{k}$ indices contribute to its commutator with $k_{\mu}$. As a result, its EOM turns out to be simplified as,

$$
\frac{d \bar{k}_{\mu}(t)}{d t}=-\int d \mathbf{k} \rho(\mathbf{k}, t) \frac{\partial \epsilon_{\mathrm{loc}}(\mathbf{k}, \overline{\mathbf{x}}, t)}{\partial \bar{x}_{\mu}}=-\frac{\partial \epsilon_{\mathrm{loc}}(\overline{\mathbf{k}}, \overline{\mathbf{x}}, t)}{\partial \bar{x}_{\mu}} .
$$

\footnotetext{
${ }^{5}$ In order to obtain Eq. 33, one has only to substitute literally Eqs. 30 into the expression for $d \overline{\mathcal{O}} / d t$ in Eqs. 29], and rename the dummy variables in the following way :

$$
\begin{aligned}
k^{\prime} & \rightarrow k_{1}, k^{\prime \prime} \rightarrow k_{2}, k \rightarrow k_{3}, \\
\sigma^{\prime} & \rightarrow m, \sigma^{\prime \prime} \rightarrow n, \sigma \rightarrow l .
\end{aligned}
$$
}


In the second equality, we replaced $\mathbf{k}$ in the integrant by its mean value, following the prescription given in Eq. (18). This is nothing but the standard EOM for the momentum of the electron wave packet shown in Eq.(11).

As for the position operator $x_{\mu}$, Eq. (23) contains both the $\mathbf{k}$-derivative and time-dependent matrix elements between different band indices. As a result, the EOM for the real space coordinate is subject to a drastic change in comparison with Eq. (2). In Sec. 2, we classified the reciprocal fields into two categories, i.e., (i) $\mathcal{F}_{k_{\mu} k_{\nu}}$, and (ii) $\mathcal{F}_{k_{\mu} t}$ and $\mathcal{F}_{k_{\mu} x_{\nu}} \frac{d \bar{x}_{\nu}}{d t}$. We will see in the next section that the decomposition (33) clearly demonstrates why we classified them in that way. We have studied on a very general basis in this section that the two components, $\Omega_{\mathcal{O}}^{(1)}$ and $\Omega_{\mathcal{O}}^{(2)}$, are structurally well distinguishable, and have completely different nature. We will see more specifically in the next section that $\Omega_{x_{\mu}}^{(1)}$ and $\Omega_{x_{\mu}}^{(2)}$ are realted respectively to the reciprocal fields (i) and (ii). Thus different origins of two types of reciprocal fields will be uncovered. The fact that the classification of reciprocal fields discussed in Sec. 2 can be done explicitly and unambiguously as the decomposition (33), is actually one of the main advantages of our approach. Let us now turn to a close inspection of the nature of $\Omega_{x_{\mu}}^{(1)}$ and $\Omega_{x_{\mu}}^{(2)}$.

\subsection{Nature of $\Omega_{x_{\mu}}^{(1)}$ and $\Omega_{x_{\mu}}^{(2)}$}

In this section let us further analyze the nature of decopmposition (33), focusing on the case of $\mathcal{O}=x_{\mu} \cdot \overline{\mathbf{x}}(t)$ is given by Eq. (16) together with Eq. (23). The first term of Eq. (B33), $\Omega_{x_{\mu}}^{(1)}$ in the present case, has particularly a familiar form, which often appears in the context of the Ehrenfest's theorem

$$
\frac{d}{d t}\left\langle\psi\left|x_{\mu}\right| \psi\right\rangle=i\left\langle\psi\left|\left[H, x_{\mu}\right]\right| \psi\right\rangle=\frac{\left\langle\psi\left|p_{\mu}\right| \psi\right\rangle}{m} .
$$

The similarity between $\Omega_{x_{\mu}}^{(1)}$ and Eq. (35) becomes clearer, when one expands the wave packet $|\psi\rangle$ in terms of a complete set of bases $\left|\phi_{\alpha}\right\rangle$ as $|\psi\rangle=\sum_{\alpha} a_{\alpha}\left|\phi_{\alpha}\right\rangle$. The difference is that the set of bases used in the expansion was complete in Eq. (35), whereas it was restricted to $N$ Bloch bands in $\Omega_{x_{\mu}}^{(1)}$. This constraint is the origin of non-vanishing field strengths.

Let us now proceed to rewrite $\Omega_{x_{\mu}}^{(1)}$ in terms of the reciprocal field strength, $\mathcal{F}_{k_{\mu} k_{\nu}}$, defined in (8). The matrix elements of the Hamiltonian, i.e., Eq. (20) in the restricted subspace, spanned by $N$ Bloch bands are calculated to be

$$
\begin{aligned}
\langle H\rangle_{m n}\left(\mathbf{k}^{\prime}, \mathbf{k}\right) & =\left\langle\phi_{m}\left(\mathbf{k}^{\prime}, \overline{\mathbf{x}}, t\right)|H| \phi_{n}(\mathbf{k}, \overline{\mathbf{x}}, t)\right\rangle=\delta\left(\mathbf{k}^{\prime}-\mathbf{k}\right)\langle H\rangle_{m n}(\mathbf{k}) \\
\langle H\rangle_{m n}(\mathbf{k}) & =\epsilon_{\mathrm{eff}}-\frac{\partial \epsilon_{\mathrm{loc}}}{\partial \bar{x}_{\nu}} \bar{x}_{\nu}+\frac{i}{2}\left\{\frac{\partial \epsilon_{\mathrm{loc}}}{\partial \bar{x}_{\nu}}\left(\nabla_{k_{\nu}}\right)_{m n}+\left(\nabla_{k_{\nu}}\right)_{m n} \frac{\partial \epsilon_{\mathrm{loc}}}{\partial \bar{x}_{\nu}}\right\}(37)
\end{aligned}
$$

where $\epsilon_{\mathrm{loc}}=\epsilon_{\mathrm{loc}}(\mathbf{k}, \overline{\mathbf{x}}, t)$ is a degenerate eigenvalue of the local Hamiltonian ${ }^{6}$. We also introduced a renormalized energy, $\epsilon_{\mathrm{eff}}(\mathbf{k}, \overline{\mathbf{x}}, t)$, which takes the form of

\footnotetext{
${ }^{6}$ See Eqs. 1214. Recall also that

$$
\left\langle H_{\mathrm{loc}}\right\rangle_{m n}\left(\mathbf{k}^{\prime}, \mathbf{k}\right)=\left\langle\phi_{m}\left(\mathbf{k}^{\prime}, \overline{\mathbf{x}}, t\right)\left|H_{\mathrm{loc}}\right| \phi_{n}(\mathbf{k}, \overline{\mathbf{x}}, t)\right\rangle=\delta\left(\mathbf{k}^{\prime}-\mathbf{k}\right) \delta_{m n} \epsilon_{\mathrm{loc}}(\mathbf{k}, \overline{\mathbf{x}}, t)
$$
}


a $N$ by $N$ matrix whose $(m, n)$-components are given by

$$
\epsilon_{m n}^{\mathrm{eff}}(\mathbf{k}, \overline{\mathbf{x}}, t)=\epsilon_{\mathrm{loc}}(\mathbf{k}, \overline{\mathbf{x}}, t) \delta_{m n}+\Delta \epsilon_{m n}(\mathbf{k}, \overline{\mathbf{x}}, t) .
$$

Its off-diagonal matrix elements are due to correction terms,

$$
\begin{aligned}
\Delta \epsilon_{m n}(\mathbf{k}, \overline{\mathbf{x}}, t) & =\frac{i}{2}\left\langle\frac{\partial u_{m}(\mathbf{k}, \overline{\mathbf{x}}, t)}{\partial k_{\mu}}\left|\left(H_{\mathrm{loc}}-\epsilon_{\mathrm{loc}}(\mathbf{k}, \overline{\mathbf{x}}, t)\right)\right| \frac{\partial u_{n}(\mathbf{k}, \overline{\mathbf{x}}, t)}{\partial \bar{x}_{\mu}}\right\rangle \\
& -\frac{i}{2}\left\langle\frac{\partial u_{m}(\mathbf{k}, \overline{\mathbf{x}}, t)}{\partial \bar{x}_{\mu}}\left|\left(H_{\mathrm{loc}}-\epsilon_{\mathrm{loc}}(\mathbf{k}, \overline{\mathbf{x}}, t)\right)\right| \frac{\partial u_{n}(\mathbf{k}, \overline{\mathbf{x}}, t)}{\partial k_{\mu}}\right\rangle
\end{aligned}
$$

where the summation over $\mu=1, \cdots, D$ was assumed implicitly. Using the matrix elements given in Eqs. (36 37) one can rewrite $\Omega_{x_{\mu}}^{(1)}$ in the following way, 7

$$
\begin{aligned}
\Omega_{x_{\mu}}^{(1)} & =\sum_{m, n=1}^{N} \int d \mathbf{k} \rho(\mathbf{k}, t) z_{m}^{*}(\mathbf{k}, t)\left\{\left[\nabla_{k_{\mu}}, \epsilon_{\mathrm{eff}}(\mathbf{k}, \overline{\mathbf{x}}, t)\right]_{m n}\right. \\
& \left.+\sum_{\nu=1}^{D}\left(\mathcal{F}_{k_{\mu} k_{\nu}}\right)_{m n} \frac{\partial \epsilon_{\mathrm{loc}}(\mathbf{k}, \overline{\mathbf{x}}, t)}{\partial \bar{x}_{\nu}}\right\} z_{n}(\mathbf{k}, t)+\Delta \Omega_{x_{\mu}}^{(1)}
\end{aligned}
$$

Apart from the energy correction $\Delta \epsilon$, the first term of $\Omega_{x_{\mu}}^{(1)}$ is nothing but a standard velocity term, i.e., the first term in the r.h.s. of Eq. (2). A remark worth mentioning here is that the covariant derivative in the commutator plays a central role in ensuring the $S U(N)$ gauge invariance of final results, which we will see later. $\Delta \Omega_{x_{\mu}}^{(1)}$ is a irrelevant term ${ }^{8}$ which vanishes with the help of prescription introduced in Eq. (18). For a later convenience, let us introduce the following abbreviated vector notation for $z_{n}(\overline{\mathbf{k}}(t), t)$ :

$$
\overline{\mathbf{z}}(t)^{\dagger} \equiv\left(z_{1}^{*}(\overline{\mathbf{k}}(t), t), \cdots, z_{N}^{*}(\overline{\mathbf{k}}(t), t)\right)
$$

where we always have in mind the prescription (18). Using this notation, one can further rewrite Eq. (39) as

$$
\Omega_{x_{\mu}}^{(1)}=\sum_{m, n=1}^{N} \bar{z}_{m}(t)^{*}\left\{\left[\nabla_{\bar{k}_{\mu}}, \epsilon_{\mathrm{eff}}(\overline{\mathbf{k}})\right]_{m n}+\sum_{\nu=1}^{D}\left(\mathcal{F}_{\bar{k}_{\mu} \bar{k}_{\nu}}\right)_{m n} \frac{\partial \epsilon_{\mathrm{loc}}(\overline{\mathbf{k}})}{\partial \bar{x}_{\nu}}\right\} \bar{z}_{n}(t)
$$

where $\overline{\mathbf{x}}$-dependence of $\epsilon_{\mathrm{loc}}(\overline{\mathbf{k}})$ is not written explicitly.

In contrast to $\Omega_{x_{\mu}}^{(1)}$, the second term of Eq. (33), $\Omega_{x_{\mu}}^{(2)}$ in the present case, would not have existed, unless the local Bloch basis had evolved in time. However, in a general situation described by a time-dependent Bloch basis, there is no reason to believe that $\Omega_{x_{\mu}}^{(2)}$ should vanish. Indeed, we will give you in Sec.

is proportional to an identity in the pseudospin space.

${ }^{7}$ Details are given in Appendix A.

${ }^{8}$ Its explicit form is given in Eq. (102) in Appendix A. 
5 some concrete examples where a finite contribution from $\Omega_{x_{\mu}}^{(2)}$ plays a crucial role in determining the physical properties of the system. Using the Eqs. (30), one can easily verify that $\Omega_{x_{\mu}}^{(2)}$ are related to the second category of reciprocal fields, i.e., $\mathcal{F}_{k_{\mu} t}$ and $\mathcal{F}_{k_{\mu} x_{\nu}} \frac{d \bar{x}_{\nu}}{d t} . \Omega_{x_{\mu}}^{(2)}$ can be rewritten as

$$
\begin{aligned}
\Omega_{x_{\mu}}^{(2)} & =-\sum_{m, n=1}^{N} \int d \mathbf{k} \rho(\mathbf{k}, t) z_{m}^{*}(\mathbf{k}, t)\left\{\left(\mathcal{F}_{k_{\mu} \bar{x}_{\nu}}\right)_{m n} \frac{d \bar{x}_{\nu}}{d t}+\left(\mathcal{F}_{k_{\mu} t}\right)_{m n}\right\} z_{n}(\mathbf{k}, t) \\
& =-\overline{\mathbf{z}}(t)^{\dagger}\left\{\sum_{\nu=1}^{D} \mathcal{F}_{\bar{k}_{\mu} \bar{x}_{\nu}} \frac{d \bar{x}_{\nu}}{d t}+\mathcal{F}_{\bar{k}_{\mu} t}\right\} \overline{\mathbf{z}}(t),
\end{aligned}
$$

where the summation over $\nu=1, \cdots, D$ was omitted in the first line. The decomposition (33) together with Eqs. (41, 42) gives a complete physical justification of the classification of $\mathcal{F}_{q_{1} q_{2}}$ done in Sec. 2. In other approaches [7, 30] the two types of reciprocal fields appear in an indistinguishable manner, and two different origins of reciprocal gauge field studied in this paper remain to be hidden.

\section{4 $S U(N)$ gauge invariance}

We have successfully related the two contributions to $\frac{d}{d t} \overline{\mathbf{x}}(t)$ in the decomposition (33), i.e., $\Omega_{x_{\mu}}^{(1)}$ and $\Omega_{x_{\mu}}^{(2)}$, respectively, to two types of gauge invariant reciprocal fields, (i) $\mathcal{F}_{k_{\mu} k_{\nu}}$, and (ii) $\mathcal{F}_{k_{\mu} t}$ and $\mathcal{F}_{k_{\mu} \underline{x}_{\nu}} \frac{d \bar{x}_{\nu}}{d t}$. Together with Eq. (34), this allows us to rewrite our EOM for $\overline{\mathbf{x}}(t)$ and $\overline{\mathbf{k}}(t)$ as

$$
\begin{aligned}
\frac{d \bar{x}_{\mu}}{d t} & =\overline{\mathbf{z}}^{\dagger}\left\{\left[\nabla_{\bar{k}_{\mu}}, \epsilon_{\mathrm{eff}}\right]-\mathcal{F}_{\bar{k}_{\mu} \bar{k}_{\nu}} \frac{d \bar{k}_{\nu}}{d t}-\mathcal{F}_{\bar{k}_{\mu} \bar{x}_{\nu}} \frac{d \bar{x}_{\nu}}{d t}-\mathcal{F}_{\bar{k}_{\mu} t}\right\} \overline{\mathbf{z}}, \\
\frac{d \bar{k}_{\mu}}{d t} & =-\frac{\partial \epsilon_{\mathrm{loc}}(\overline{\mathbf{k}}, \overline{\mathbf{x}}, t)}{\partial \bar{x}_{\mu}} .
\end{aligned}
$$

Repeated indices $\nu$ should be summed over $\nu=1, \cdots, D$. The effective energy $\epsilon_{\text {eff }}$ is related to the local $\epsilon_{\text {loc }}$ as Eq. (38). In Eqs. (4344), $\epsilon_{\text {eff }}$ and $\epsilon_{\text {loc }}$ are functions of $\overline{\mathbf{k}}, \overline{\mathbf{x}}, t$, i.e., $\epsilon_{\mathrm{eff}}=\epsilon_{\mathrm{eff}}(\overline{\mathbf{k}}, \overline{\mathbf{x}}, t), \epsilon_{\mathrm{loc}}=\epsilon_{\mathrm{loc}}(\overline{\mathbf{k}}, \overline{\mathbf{x}}, t)$. Below let us verify explicitly that Eqs. (43) are indeed $S U(N)$ gauge invariant. In order to obtain a complete set of EOM, however, we still need to know an EOM for $\overline{\mathbf{z}}(t)$ defined in (40). The details of its derivation is given in Appendix B, and the result is,

$$
i \frac{d \overline{\mathbf{z}}}{d t}=\left(\epsilon_{\mathrm{eff}}-\bar{x}_{\mu} \frac{\partial \epsilon_{\mathrm{loc}}}{\partial \bar{x}_{\mu}} \mathbf{1}-\frac{d \bar{k}_{\mu}}{d t} \mathcal{A}_{\bar{k}_{\mu}}-\frac{d \bar{x}_{\mu}}{d t} \mathcal{A}_{\bar{x}_{\mu}}-\mathcal{A}_{t}\right) \overline{\mathbf{z}}
$$

where repeated indices $\mu$ should be summed over $\mu=1, \cdots, N$, and again, $\epsilon_{\text {eff }}=\epsilon_{\text {eff }}(\overline{\mathbf{k}}, \overline{\mathbf{x}}, t), \epsilon_{\text {loc }}=\epsilon_{\text {loc }}(\overline{\mathbf{k}}, \overline{\mathbf{x}}, t)$. Eqs. 434445) consitute the central result of this paper together with their interpretation in terms of the field strength $\mathcal{F}_{q_{1} q_{2}}$, which will be further discussed in Sec. 5 .

Before turning to the discussion on gauge invariance, let us make a few comments on Eq. (45). On the r.h.s. the diagonal part of $\epsilon_{\text {eff }}$, i.e., $\epsilon_{\text {loc }}(\overline{\mathbf{k}}) \mathbf{1}$, 
tpgether with the second term simply give rise to a usual $U(1)$ phase factor associated with an effective energy, $\epsilon_{\text {loc }}(\overline{\mathbf{k}}, \overline{\mathbf{x}}, t)-\sum_{\mu=1}^{N} \bar{x}_{\mu} \frac{\partial \epsilon_{\mathrm{loc}}(\overline{\mathbf{k}}, \overline{\mathbf{x}}, t)}{\partial \bar{x}_{\mu}}$. On the other hand, $\Delta \epsilon(\mathbf{k})$ generally has off-diagonal matrix elements between different bands and thereby yields a nontrivial $S U(N)$ phase factor, which corresponds to the precession of the spin and/or orbital associated with the wave packet. The remaining terms of Eq. (45) represents a Berry-Wilczek-Zee phase [27] originating from the adibatic motion of the wave packet. The first two terms are due to its motion in $(\overline{\mathbf{k}}, \overline{\mathbf{x}})$-space. In the Abelian case $(N=1)$, the EOM for $\overline{\mathbf{x}}(t)$ and $\overline{\mathbf{k}}(t)$, i.e., Eqs. (4344), are independent of the motion of phase degree of freedom, $\overline{\mathbf{z}}(t)$, whereas $\overline{\mathbf{z}}(t)$ acquires a quantal phase due to the evolution of $\overline{\mathbf{x}}$ and $\overline{\mathbf{k}}: \exp \left[i \int d t\left(\frac{d \bar{k}_{\mu}}{d t} \mathcal{A}_{\bar{k}_{\mu}}+\frac{d \bar{x}_{\mu}}{d t} \mathcal{A}_{\bar{x}_{\mu}}+\mathcal{A}_{t}\right)\right]$ where the summation over $\mu$ was omitted. This is analogous to the Berry quantal phase [16].

Since the $N$-fold Bloch states are energetically degenerate over the whole Brillouin zone, these EOM should be independent of the choice of $N$ Bloch bases and be invariant under the following gauge transformation :

$$
\begin{aligned}
\left|\tilde{u}_{n}(\overline{\mathbf{k}}, \overline{\mathbf{x}}, t)\right\rangle & =\sum_{m=1}^{N}\left|u_{m}(\overline{\mathbf{k}}, \overline{\mathbf{x}}, t)\right\rangle g_{m n}(\overline{\mathbf{k}}, \overline{\mathbf{x}}, t) \\
\tilde{\mathbf{z}}(\overline{\mathbf{k}}, t) & =g^{-1}(\overline{\mathbf{k}}, \overline{\mathbf{x}}, t) \mathbf{z}(\overline{\mathbf{k}}, t) .
\end{aligned}
$$

Here $\left|u_{n}(\overline{\mathbf{k}}, \overline{\mathbf{x}}, t)\right\rangle$ and $\mathbf{z}(\overline{\mathbf{k}}, t)$ are transformed inversely to each other, making the l.h.s. of Eq. (15) invariant. The gauge field and the field strength associated with it are transformed in the following way,

$$
\begin{aligned}
\tilde{\mathcal{A}}_{q_{\mu}} & =g^{-1} \mathcal{A}_{q_{\mu}} g-i g^{-1} \frac{\partial g}{\partial q_{\mu}}, \\
\tilde{\mathcal{F}}_{q_{\mu} q_{\nu}} & =g^{-1} \mathcal{F}_{q_{\mu} q_{\nu}} g,
\end{aligned}
$$

where $q_{\mu}=\overline{\mathbf{k}}, \overline{\mathbf{x}}, t$. Concomitantly, the covariant derivative, defined generally for this $q_{\mu}$ as

$$
\nabla_{q_{\mu}}=\frac{\partial}{\partial q_{\mu}}-i \mathcal{A}_{q_{\mu}}
$$

obeys the following transformation rule:

$$
\tilde{\nabla}_{q_{\mu}}=g^{-1} \nabla_{q_{\mu}} g .
$$

Futhermore, one can also check that the $N \times N$ matrix $\epsilon_{\text {eff }}(\overline{\mathbf{k}})$ is transformed as

$$
\tilde{\epsilon}_{\mathrm{eff}}(\overline{\mathbf{k}})=g^{-1} \epsilon_{\mathrm{eff}}(\overline{\mathbf{k}}) g .
$$

Using the above transformation rules, one can indeed verify that our EOM 43 44) are invariant under $S U(N)$ gauge transformation (46). 


\subsection{Abelian case : comparison with other approaches}

In the Abelian case : $N=1$, the above equations of motion (43)44) reduces to

$$
\begin{aligned}
\frac{d \bar{x}_{\mu}}{d t} & =\frac{\partial \epsilon_{\mathrm{eff}}(\overline{\mathbf{k}}, \overline{\mathbf{x}}, t)}{\partial \bar{k}_{\mu}}-\mathcal{F}_{\bar{k}_{\mu} \bar{k}_{\nu}} \frac{d \bar{k}_{\nu}}{d t}-\mathcal{F}_{\bar{k}_{\mu} \bar{x}_{\nu}} \frac{d \bar{x}_{\nu}}{d t}-\mathcal{F}_{\bar{k}_{\mu} t} \\
\frac{d \bar{k}_{\mu}}{d t} & =-\frac{\partial \epsilon_{\mathrm{loc}}(\overline{\mathbf{k}}, \overline{\mathbf{x}}, t)}{\partial \bar{x}_{\mu}}
\end{aligned}
$$

EOM similar to Eqs. (4950) have been derived, to our knowledge, twice, using either

1. Time-dependent variational principle [7] or,

2. Path-integral method using Wannier basis. ${ }^{9}$

If we compare Eqs. (2.19) of Ref. [7] and our Eqs. (4950), it can be observed that three terms,

$$
\mathcal{F}_{\bar{x}_{\mu} \bar{x}_{\nu}} \frac{d \bar{x}_{\nu}}{d t}+\mathcal{F}_{\bar{x}_{\mu} \bar{k}_{\nu}} \frac{d \bar{k}_{\nu}}{d t}+\mathcal{F}_{\bar{x}_{\mu} t}
$$

are lacking on the right hand side of (150). However, one can easily check by a simple power counting that these terms appear only at orders higher than 2 in the perturbation series w.r.t. $\beta$ or $\mathbf{x}-\overline{\mathbf{x}}$. Let us briefly illustrate this point. Since a subscript $\bar{x}$ implies a derivative w.r.t. $\bar{x}$, which is always accompanied by $\mathbf{x}-\overline{\mathbf{x}}$, it increases the power by one. It is also the case for the subscript $t$. Therefore, the first and the last terms of (50) turs out immediately to be at least of the second order of $\beta$. In the second line, i.e., in Eq. (50), since $\partial \epsilon_{\mathrm{loc}}(\overline{\mathbf{k}}, \overline{\mathbf{x}}, t) / \partial \bar{x}_{\mu}$ is also of the first order w.r.t. $\beta$, one can verify that $d \bar{k}_{\mu} / d t$ is at least of the first order w.r.t. $\beta$, even if (51) is added to it. Taking everything into account, one can conclude that the lacking terms (51) are at least of the second order w.r.t. $\beta$ or $\mathbf{x}-\overline{\mathbf{x}}$.

Another difference between Eqs. (4950) and Eqs. (2.19) of Ref. [7] is that in our EOM for $d \overline{\mathbf{k}} / d t$, the derivative $\partial / \partial \bar{x}_{\mu}$ applies to $\epsilon_{\mathrm{loc}}(\overline{\mathbf{k}}, \overline{\mathbf{x}}, t)$ and not to $\epsilon_{\mathrm{eff}}(\overline{\mathbf{k}}, \overline{\mathbf{x}}, t)$ as in Ref. [7. In our formalism, as is clearly shown in Eq. (34), there is no room for $\Delta \epsilon(\overline{\mathbf{k}}, \overline{\mathbf{x}}, t)$ to enter the expression (50). Nevertheless, repeating the same type argument, i.e., the power counting for $\Delta \epsilon(\overline{\mathbf{k}}, \overline{\mathbf{x}}, t)$, one can confirm that the contribution from $\Delta \epsilon(\overline{\mathbf{k}}, \overline{\mathbf{x}}, t)$ is not physically relevant at the first order of $\beta$ or of $\mathbf{x}-\overline{\mathbf{x}}$.

We have not only developed a systematic perturbation theory w.r.t. $\beta$ or $\mathbf{x}-\overline{\mathbf{x}}$, but also we make no approximation apart from the assumptions stated in Sec. 3. Our calculation must be, therefore, exact at the first order of perturbation theory. Since possible discrepancies start only at the second order in

\footnotetext{
${ }^{9}$ We had some difficulty to jusitify the use of Wannier basis used in Ref. [30] as a complete basis necessary in the path integral formalism. This is closely related to the arbitrariness of Wannier function discussed extensively in Ref. 34]
} 
the perturbation series, our result, Eq. (4950) is not inconsistent ${ }^{10}$ with that of Ref. [7.

\section{Discussion : Berry phase engineering}

The gauge invariant EOM (4344) have been successfully derived in the previous section. The decomposition (33) uncovered the orgin of two different types of reciprocal fields introduced in Sec. 2. In this section we discuss some physical consequences of Sec. 4 in the context of Berry phase engineering.

In the Introduction, we argued that a finite net charge current could be induced by the $U(1)$ Berry phase correction to the semiclassical EOM (12). This finite charge current is actually carried by all the electrons below the Fermi surface, i.e., by the electrons in the ground state. Generalizing this $U(1)$ argument to the non-Abelian case, we will discuss in this section how the various types of non-abelian field strength appearing in our EOM are related to concrete physical realizations, mainly focusing on the $S U(2)$ case. This opens a new possibility of manipulating the ground state electronic wave function by controlling Berry phase, which is sometimes called, Berry phase engineering.

After introducing some terminologies and fixing notations, we will focus on two topics. In Sec. 5.2, we will see that $\mathcal{F}_{k_{\mu} k_{\nu}}$ is related to the physics of Hall type current. We first observe that the charge Hall current can be described by a trace of non-abelian field strength $\mathcal{F}_{k_{\mu} k_{\nu}}$, while this current vanishes whenever the system is time-reversally ( $T$-) invariant. The charge Hall current carried by a $(\mathbf{k}, \uparrow)$ Bloch electron and that of the $(-\mathbf{k}, \downarrow)$ electron precisely cancel each other. Based on this observation, we then propose two physical situations in which the difficulty of the cancellation of Hall type charge current will be overcome and the reciprocal magnetic field manifests itself experimentally as a Hall type current. The two situations are :

1. anomalous (charge) Hall current observed in systems with broken reversal symmetry, i.e., in ferromagnets, 6, 23, 24, 25]

2. spin Hall current in time reversally symmetric systems. 8, 9, 10, 11, 12,

In Sec. 5.3, we will argue that $\mathcal{F}_{k_{\mu} t}$ is directly related to various types of polarization currents, currents induced in insulators under time-dependent perturbations. Similarly to the case of Hall type charge current, which will be discussed in Sec. 5.2, we first observe that in such systems that are symmetric under spatial inversion, the polarization electric/spin current actually vanishes due to a cancellation associated with the inversion symmetry of the system. Then, in order to overcome this difficulty we propose, in parallel with Sec. 5.2, two physical systems in which the problem of cancellation will be resolved, and

\footnotetext{
${ }^{10}$ The calculation done in Ref. 7 is also first order, in particular, their Hamiltonian, Eqs. (2.1), (2.14) and (2.15), is first order, but they kept all the possible Berry phase contribution without making further consideration of power counting, whereas we omitted systematically higher order terms.
} 
the gauge invariant reciprocal field strength appears explicitly in a macroscopic physcial quantity, i.e., as a polarization current:

1. If the inversion symmetry is broken externally or spontaneously, the abelian $\mathcal{F}_{k_{\mu} t}$ gives rise to a relevant contribution to the polarization electric/spin current.

2. Even in systems symmetric under spatial inversion, non-abelian $\mathcal{F}_{k_{\mu} t}$ may have a chance to manifest itself as a polarization orbital current, if that orbital degree of freedom changes its sign under the spatial inversion.

The analogy and correspondence between the Hall type and polarization currents are summarized in Tables 1 and 2 .

\subsection{Preliminaries}

Before further discussing Berry phase transport, we first introduce some terminologies as well as giving an unambiguous definition to spin/orbital currents.

In order to illustrate our point, let us first consider a spin current. We have naturally in mind that there are different points of view [10, 11, 12] on the definition of spin current operator, $J_{\alpha \mu}^{\mathcal{S}}$. The difficulty of defininig a spin current stems simply from the fact that the bare spin $S_{\alpha}$ is generally not a conserved quantity due to spin-orbit interaction, i.e., the continuity eqation, $\partial S_{\alpha} / \partial t+$ $\sum_{\mu=1}^{D} \partial J_{\mu \alpha}^{\mathcal{S}} / \partial x_{\mu}=0$, is not satisfied. Thereby the Noether's theorem does not apply. If one focuses on the time derivative of a local spin $S_{\alpha}(\mathbf{x}, t)$ in the general case of non-conserved spin, one could observe that there are two contributions to it of physically different nature, i.e., contributions from (i) a local spin curent, (ii) a local precession of spin. The former is the one of our interest, and the latter is related to the non-conservation of spin. Unfortunately, there is no systematic prescription for distinguishing between those two contributions.

We can still define on quite general ground a current operator $\mathbf{J}_{\mathcal{I}}$ associated with an internal degree of freedom $\mathcal{I}$ as the time derivative of a spatial polarization of $\mathcal{I}$ as,

$$
\begin{aligned}
\mathbf{J}_{\mathcal{I}} & =\frac{d \mathbf{P}_{\mathcal{I}}}{d t} \\
\mathbf{P}_{\mathcal{I}} & =\frac{1}{L^{D}} \sum_{j=1}^{M} \frac{1}{2}\left\{\mathcal{I}_{j} \mathbf{x}_{j}+\mathbf{x}_{j} \mathcal{I}_{j}\right\} .
\end{aligned}
$$

$L$ and $M$ denote the system size and the total number of electrons, respectively. The subscripts $j$ attributed to $\mathcal{I}$ and $\mathbf{x}$ specify each electron. In the case of a spin current, the operator $\mathcal{I}$ should be replaced by a usual spin operator, $\mathcal{I}=S_{\alpha}$. The main reason why we have defined the spin/orbital current as 
Eq. (5253) is that that is the one which could be diretcly observable. ${ }^{11}$ For example, an increasing $\left(P_{S_{\alpha}}\right)_{\mu}=P_{\mu}\left(S_{\alpha}\right)$ indicates that extra up (down)-spin electrons with $S_{\alpha}=+1 / 2(-1 / 2)$ acculmulate in one (the other) end of a system with $x_{\mu}=L / 2(-L / 2)$, which could be experimentally detected by some optical probes.

We will also discuss orbital currents. A Bloch electron has, in addition to the spin degree of freedom, orbital degrees of freedom $\Pi$ in multiband systems. These orbital degrees of freedom describe the charge distribution in the unit cell, and hence, ${ }^{12}$

$$
\Pi(\mathbf{x}, \mathbf{p})=\Pi(\mathbf{x}+\mathbf{a}, \mathbf{p}) .
$$

In Sec. 5.3 we focus on an orbital operator $\Pi$ which behaves quite contrastingly to the spin under time reversal and spatial inversion, i.e.,

$$
\begin{aligned}
& \Pi(\mathbf{x}, \mathbf{p})=-\Pi(-\mathbf{x},-\mathbf{p}) \\
& \Pi(\mathbf{x}, \mathbf{p})=\Pi^{*}(\mathbf{x}, \mathbf{p}) .
\end{aligned}
$$

In contrast to the spin operator $S$, the parity operator $\Pi$ reverses its sign under spatial inversion, while invariant under time reversal. Accordingly, we dub this oribtal operator as a parity operator. ${ }^{13}$ Various transformation properties of the operators $\mathcal{I}=S_{\alpha}, \Pi$ are summarized in Table 1 .

The spin/orbital current associated with an operator $\mathcal{I}$ was introduced in Eqs. (5253). We calculate in Secs. 5.2 and 5.3 those spin/parity currents carried by the ground state. In order to make later discussions clearer and physically more appealing, we make the following assumptions:

1. We keep only their matrix elements in the restricted subspace $\mathcal{N}$ spanned by $N$-fold degenerate bands ; If $m \in \mathcal{N}$ and $l \notin \mathcal{N}$, then

$$
\begin{aligned}
\left\langle\phi_{m}(\mathbf{k})\left|S_{\alpha}\right| \phi_{l}(\mathbf{k})\right\rangle & =0, \\
\left\langle\phi_{m}(\mathbf{k})|\Pi(\mathbf{x}, \mathbf{p})| \phi_{l}(\mathbf{k})\right\rangle & =0 .
\end{aligned}
$$

${ }^{11}$ Otherwise, we could have defined it also as

$$
\tilde{\mathbf{J}}_{\mathcal{I}}=\frac{1}{L^{D}} \sum_{j=1}^{M} \frac{1}{2}\left\{\mathcal{I}_{j} \frac{d \mathbf{x}}{d t}+\frac{d \mathbf{x}}{d t} \mathcal{I}_{j}\right\} .
$$

This type of definition is convenient for the application of Kubo formula. 12,11$] 29$

${ }^{12}$ The periodicity of $\Pi$, i.e., Eq. [55] excludes its off-diagonal matrix elements in k-space:

$$
\begin{aligned}
& \langle\Pi\rangle_{m n}\left(\mathbf{k}^{\prime}, \mathbf{k}\right)=\delta\left(\mathbf{k}^{\prime}-\mathbf{k}\right)\langle\Pi\rangle_{m n}(\mathbf{k}) \\
& \langle\Pi\rangle_{m n}(\mathbf{k})=\left\langle\phi_{m}(\mathbf{k}, t)|\Pi| \phi_{n}(\mathbf{k}, t)\right\rangle \\
& =\frac{(2 \pi)^{D}}{V_{\text {cell }}} \int_{\text {unit cell }} d \mathbf{x}\left\langle u_{m}(\mathbf{k}, t) \mid \mathbf{x}\right\rangle \Pi(\mathbf{x},-i \nabla+\mathbf{k})\left\langle\mathbf{x} \mid u_{n}(\mathbf{k}, t)\right\rangle .
\end{aligned}
$$

\footnotetext{
${ }^{13}$ This orbital operator is different from the angular momentum operator, $\omega=\mathbf{x} \times \mathbf{p}$, which we might also call an orbital operator. $\omega$ reverses its sign under time reversal, while remains invariant under spatial inversion.
} 
As will be seen in Secs. 5.2 and 5.3, these approximations make the following discussions considerably simpler, i.e., not only a charge current but also spin/parity currents become related simply to the non-abelian field strength, $\mathcal{F}_{k_{\mu} k_{\nu}}$ and $\mathcal{F}_{k_{\mu} t}$.

\section{$5.2 \mathcal{F}_{k_{\mu} k_{\nu}}$ induces Hall type currents: AHE and spin Hall effect}

The field strength $\mathcal{F}_{k_{\mu} k_{\nu}}$ describes various kinds of spontaneous Hall currents carried by the ground state. In order to demonstrate it we expose our system under a uniform electric field $\mathbf{E}$; we consider below the following Hamiltonian,

$$
H(\mathbf{p}, \mathbf{x}, \beta(\mathbf{x}, t))=H_{0}(\mathbf{p}, \mathbf{x})+e \sum_{\mu=1}^{D} E_{\mu} x_{\mu} .
$$

We see below that the Hall-type current can be expressed essentially as a trace of $\mathcal{I F}_{k_{\mu} k_{\nu}}$ in the $N$-fold degenerate pseudospin space. The cancellation or the survival of such Hall-type topological current is essentially related to the transformation properties under time reversal $(T)$ of the system. In systems with broken $T$ symmetry a finite charge/mass Hall current generally appear. In two spatial dimension $D=2$, this situation is often described in terms of ChernSimons gauge field, accounting for the quantized Hall conductance, 35] as well as fractional charge and statistics. [36] Chern-Simons terms also appear in electrically neutral systems. [37] Quantization of spin Hall conductance in unconventional superfluids has also been studied in this context. 38.

\subsubsection{Charge Hall current}

Let us first see that the trace of the non-abelian field strength $\mathcal{F}_{k_{\mu} k_{\nu}}$ describes a spontaneous charge Hall conductivity, i.e., an anomalous Hall conductivity. 66, 23, 24, 25] In terms of Eqs. (52 53), we are considering the case of $\mathcal{I}=\mathbf{1}$. Applying the EOM, Eqs. (43144), to the present case, we consider a Bloch electron, in the $j$-th pseudospin state ${ }^{14}$ and with a crystal momentum $\overline{\mathbf{k}}$. The $S U(N)$ gauge invariant EOM for this electron under a uniform electric field $\mathbf{E}$ now reads,

$$
\begin{aligned}
\frac{d \bar{x}_{\mu}^{(j)}}{d t} & =\frac{\partial \epsilon_{\text {loc }}(\overline{\mathbf{k}})}{\partial \bar{k}_{\mu}}-\sum_{\nu=1}^{D} \mathbf{z}^{(j) \dagger} \mathcal{F}_{k_{\mu} k_{\nu}}(\overline{\mathbf{k}}) \mathbf{z}^{(j)} \frac{d \bar{k}_{\nu}}{d t}, \\
\frac{d \bar{k}_{\mu}}{d t} & =-e E_{\mu} .
\end{aligned}
$$

When the crystal momentum $\overline{\mathbf{k}}$ is located below the Fermi surface, all those pseudospin states are completely occupied and contribute to the charge current.

\footnotetext{
${ }^{14}$ The $j$-th eiegenstate is a linear combination of different pseudospin states $m=1, \cdots, N$, i.e., $\sum_{m=1}^{N} z_{m}^{(j)}\left|\phi_{m}(\overline{\mathbf{k}})\right\rangle$. These eigenstates are chosen to be orthogonal to each other: $\mathbf{z}^{(i) \dagger} \mathbf{z}^{(j)}=\delta_{i j}$ for $i, j=1, \cdots, N$.
} 
In order to calculate the charge Hall current, we need

$$
\begin{aligned}
-e \sum_{j=1}^{N} \frac{d \bar{x}_{\mu}^{(j)}}{d t} & =-N e \frac{\partial \epsilon_{\mathrm{loc}}(\overline{\mathbf{k}})}{\partial \bar{k}_{\mu}}-e^{2} \sum_{j=1}^{N} \mathbf{z}^{(j) \dagger} \mathcal{F}_{k_{\mu} k_{\nu}}(\overline{\mathbf{k}}) \mathbf{z}^{(j)} E_{\nu} \\
& =-N e \frac{\partial \epsilon_{\mathrm{loc}}(\overline{\mathbf{k}})}{\partial \bar{k}_{\mu}}-e^{2} \operatorname{Tr}\left[\mathcal{F}_{k_{\mu} k_{\nu}}(\overline{\mathbf{k}})\right] E_{\nu} .
\end{aligned}
$$

Then, by integrating these contributions over filled $\overline{\mathbf{k}}$ points, we obtain the total current carried by all the electrons below the Fermi energy $\epsilon_{\mathrm{F}}$,

$$
\left(J_{\mathcal{C}}^{\text {Hall }}\right)_{\mu}=-e^{2} \sum_{\nu=1}^{D} \int_{\epsilon_{\text {loc }}(\overline{\mathbf{k}})<\epsilon_{\mathrm{F}}} \frac{d \overline{\mathbf{k}}}{(2 \pi)^{D}} \operatorname{Tr}\left[\mathcal{F}_{k_{\mu} k_{\nu}}(\overline{\mathbf{k}})\right] E_{\nu} .
$$

Here we assumed for the sake of simplicity that the non-perturbed Hamiltonian $H_{0}(\mathbf{p}, \mathbf{x})$ is also invariant under spatial inversion $(I)$. As far as the Hall type current is concerned, however, this $I$ symmetry plays only a minor role. Eq. (64) takes indeed the form of a Hall type current reflecting the antisymmetry of $\mathcal{F}_{k_{\mu} k_{\nu}}: \mathcal{F}_{k_{\mu} k_{\nu}}=-\mathcal{F}_{k_{\nu} k_{\mu}}$. The first term of Eq. (63) did not contribute to Eq. (64) due to a cancellation associated with the $I$ symmetry. On the contrary, Eq. (64) remains finite irrespective of the $I$-invariance of $H_{0}$.

Unfortunately, the charge Hall current obtained in Eq. (64) vanishes whenever the system is $T$-invariant. Let us see this point more explicitly. We consider an unperturbed Hamiltonian $H_{0}(\mathbf{x}, \mathbf{p})$ which is invariant under $T$. The $T$-invariance relates its $N$-fold degenerate Bloch functions at $\mathbf{k}$ with those at - k upto a certain $S U(N)$ gauge transformations $g(\mathbf{k})$,

$$
\sum_{b=\uparrow, \downarrow}\left[i \sigma_{y}\right]_{a b}\left\langle\mathbf{x}, b \mid u_{i}(-\mathbf{k})\right\rangle^{*}=\sum_{j=1}^{N}\left\langle\mathbf{x}, a \mid u_{j}(\mathbf{k})\right\rangle g_{j i}(\mathbf{k}) .
$$

In the language of field strength, this reduces to,

$$
\mathcal{F}_{k_{\mu} k_{\nu}}(-\mathbf{k})^{t}=-g^{-1} \mathcal{F}_{k_{\mu} k_{\nu}}(\mathbf{k}) g,
$$

where the superscript $t$ represents a transposed matrix, i.e., $\left(\mathcal{F}^{t}\right)_{m n}=(\mathcal{F})_{n m}$. One can verify this using Eqs. (7865). Consequently, the charge current carried by a Bloch electron at $\mathbf{k}$ cancels with that of $\mathbf{- k}$ electron, i.e.,

$$
\sum_{\nu=1}^{D} \operatorname{Tr}\left[\mathcal{F}_{k_{\mu} k_{\nu}}(\mathbf{k})\right] E_{\nu}=-\sum_{\nu=1}^{D} \operatorname{Tr}\left[\mathcal{F}_{k_{\mu} k_{\nu}}(-\mathbf{k})\right] E_{\nu} .
$$

Eqs. (6467) indicate the absence of spontaneous Hall current in $T$-invariant systems.

\subsubsection{Spin Hall current}

We are thus led to investigate the spin current, expecting that the spin Hall current remains finite even in $T$-invariant systems. [8, 9, 10, 11, 12] The 
underlying idea is simply that such a sign change as seen in Eq. (67) may be compensated by that of spin operator under the operation of time reversal. The spin current has been defined in Eq. (52), in a more general context for an arbitrary internal degree of freedom $\mathcal{I}$. Let us first observe that the $T$-invariance of $H_{0}(\mathbf{x}, \mathbf{p})$ is instrumental for this compensation. The total spin carried by the Bloch electrons at $\mathbf{k}$ has the same absolute value and opposite sign of that of the Bloch electrons at $-\mathbf{k},{ }^{15}$

$$
\operatorname{Tr}\left[\left\langle S_{\alpha}\right\rangle(\mathbf{k})\right]=-\operatorname{Tr}\left[\left\langle S_{\alpha}\right\rangle(-\mathbf{k})\right],
$$

where $\left\langle S_{\alpha}\right\rangle(\mathbf{k})$ is a $N \times N$ matrix, whose $(m, n)$-components are given by

$$
\begin{aligned}
& \left\langle S_{\alpha}\right\rangle_{m n}(\mathbf{k})=\left\langle\phi_{m}(\mathbf{k})\left|S_{\alpha}\right| \phi_{n}(\mathbf{k})\right\rangle \\
= & \frac{(2 \pi)^{D}}{V_{\text {cell }}} \int_{\text {unit cell }} d \mathbf{x} \sum_{a, b=\uparrow, \downarrow}\left\langle u_{m}(\mathbf{k}) \mid \mathbf{x}, a\right\rangle \frac{1}{2}\left(\sigma_{\alpha}\right)_{a b}\left\langle\mathbf{x}, b \mid u_{n}(\mathbf{k})\right\rangle .
\end{aligned}
$$

Let us now consider the spin Hall current defined as (5253) with $\mathcal{I}$ being $\mathcal{I}=S_{\alpha}$, the usual spin operator. In order to evaluate a spin Hall current, the EOM (6162) used for the calculation of charge Hall current are no longer sufficient. We need instead to derive EOM for an observable,

$$
\mathcal{O}_{\mu \alpha}^{\mathcal{S}}=\mathcal{O}_{\mu}\left(S_{\alpha}\right)=\frac{1}{2}\left(S_{\alpha} x_{\mu}+x_{\mu} S_{\alpha}\right)
$$

Following the same type of procedure as the derivation of Eqs. 6162), we perform, in particular, the decomposition (33). As was also the case in Eqs. [6162), the second contribution to [33), i.e., $\Omega_{\mathcal{O}}^{(2)}$ vanishes in the present case.

16 The EOM reads,

$$
\begin{aligned}
& \frac{d \overline{\mathcal{O}}_{\mu \alpha}^{\mathcal{S}}}{d t}=i \sum_{m, n=1}^{N} \int d \mathbf{k} d \mathbf{k}^{\prime} a_{m}^{*}(\mathbf{k}, t)\left[\langle H\rangle,\left\langle\mathcal{O}_{\mu \alpha}^{\mathcal{S}}\right\rangle\right]_{m n}\left(\mathbf{k}, \mathbf{k}^{\prime}\right) a_{n}\left(\mathbf{k}^{\prime}, t\right) \\
& =i \sum_{m, n=1}^{N} \int d \mathbf{k} a_{m}^{*}\left[\left\langle H_{0}\right\rangle+e \sum_{\mu=1}^{D} E_{\mu}\left\langle x_{\mu}\right\rangle,\left\langle\mathcal{O}_{\mu \alpha}^{\mathcal{S}}\right\rangle\right]_{m n} a_{n} .
\end{aligned}
$$

The assumption (58) allows us to rewrite Eq. (172) as

$$
\begin{aligned}
& \frac{d \overline{\mathcal{O}}_{\mu \alpha}^{\mathcal{S}}}{d t}=\sum_{m, n=1}^{N} \int d \mathbf{k} \rho(\mathbf{k}) z_{m}^{*}(\mathbf{k}, t)\left[\left\langle S_{\alpha}\right\rangle_{m n}(\mathbf{k}) \frac{\partial \epsilon_{\mathrm{loc}}(\overline{\mathbf{k}})}{\partial k_{\nu}}\right. \\
& \left.+\frac{e}{2} \sum_{\nu=1}^{D}\left\{\left(\left\langle S_{\alpha}\right\rangle(\mathbf{k}) \mathcal{F}_{k_{\mu} k_{\nu}}(\mathbf{k})\right)_{m n} E_{\nu}+\text { h.c. }\right\}\right] z_{n}(\mathbf{k}, t) .
\end{aligned}
$$

\footnotetext{
${ }^{15}$ To be more specific, $T$-invariance relates the matrix of $S_{\alpha}$ at $\mathbf{k}$ and at $-\mathbf{k}$ through a $S U(N)$ gauge transformation $g$,

$$
\left\langle S_{\alpha}\right\rangle(-\mathbf{k})^{t}=-g^{-1}\left\langle S_{\alpha}\right\rangle(\mathbf{k}) g .
$$

Eq. 68 can be verified explicitly using Eq. 65.

${ }^{16} \mathrm{As}$ long as the electric field $\mathbf{E}$ in Eq. (60) is uniform, the local Bloch function defined in Eq. (12) has no dependence on $\overline{\mathbf{x}}$ and $t$. As a result, $\Omega_{\mathcal{O}}^{(2)}$ vanishes.
} 
The details of the derivation of Eq. (73) is given in Appendix C. We then apply the prescription (18), replacing $\mathbf{k}$ in the integrant by its mean value $\overline{\mathbf{k}}$. The contribution to the spin Hall current by an electron occupying the $j$-th pseudospin state at $\overline{\mathbf{k}}$ is, therfore,

$$
\begin{aligned}
\frac{d \overline{\mathcal{O}}_{\mu \alpha}^{\mathcal{S}(j)}}{d t} & =\mathbf{z}^{(j) \dagger}\left\langle S_{\alpha}\right\rangle(\overline{\mathbf{k}}) \mathbf{z}^{(j)} \frac{\partial \epsilon_{\text {loc }}(\overline{\mathbf{k}})}{\partial \bar{k}_{\mu}} \\
& +\frac{e}{2} \sum_{\nu=1}^{D}\left\{\mathbf{z}^{(j) \dagger}\left\langle S_{\alpha}\right\rangle(\overline{\mathbf{k}}) \mathcal{F}_{k_{\mu} k_{\nu}}(\overline{\mathbf{k}}) \mathbf{z}^{(j)}+\text { c.c. }\right\} E_{\nu} .
\end{aligned}
$$

Finally we take the summation over $N$ pseudospin states and over filled $\overline{\mathbf{k}}$ points to find,

$$
\begin{aligned}
\left(J_{\mathcal{S}}^{\text {Hall }}\right)_{\mu \alpha} & =\int_{\epsilon_{\mathrm{loc}}(\overline{\mathbf{k}})<\epsilon_{\mathrm{F}}} \frac{d \overline{\mathbf{k}}}{(2 \pi)^{D}} \sum_{j=1}^{N} \frac{d \overline{\mathcal{O}}_{\mu \alpha}^{\mathcal{S}(j)}}{d t} \\
& =e \sum_{\nu=1}^{D} \int_{\epsilon_{\mathrm{loc}}(\overline{\mathbf{k}})<\epsilon_{\mathrm{F}}} \frac{d \overline{\mathbf{k}}}{(2 \pi)^{D}} \operatorname{Tr}\left[\left\langle S_{\alpha}\right\rangle(\overline{\mathbf{k}}) \mathcal{F}_{k_{\mu} k_{\nu}}(\overline{\mathbf{k}})\right] E_{\nu}
\end{aligned}
$$

As was the case in Eq. (64), we also assumed in Eq. (75) that $H_{0}$ is invariant under I, so that the first term of Eq. (74) should not appear in Eq. (75), i.e.,

$$
\int_{\epsilon_{\mathrm{loc}}(\overline{\mathbf{k}})<\epsilon_{\mathrm{F}}} \frac{d \overline{\mathbf{k}}}{(2 \pi)^{D}} \operatorname{Tr}\left[\left\langle S_{\alpha}\right\rangle(\mathbf{k})\right] \frac{\partial \epsilon_{\mathrm{loc}}(\overline{\mathbf{k}})}{\partial \bar{k}_{\nu}}=0 .
$$

Contrary to this normal part, the spin Hall current associated with the anomalous velocity, given by Eq. (75), has a possibility to be finite irrespective of the $I$ and $T$ symmetries. When the system is invariant under either $T$ or $I,{ }^{17}$ the spin current carried by a Bloch electron at $\mathbf{k}$ and that of $-\mathbf{k}$ give the same contribution;

$$
\operatorname{Tr}\left[\left\langle S_{\alpha}\right\rangle(\mathbf{k}) \mathcal{F}_{k_{\mu} k_{\nu}}(\mathbf{k})\right]=\operatorname{Tr}\left[\left\langle S_{\alpha}\right\rangle(-\mathbf{k}) \mathcal{F}_{k_{\mu} k_{\nu}}(-\mathbf{k})\right] .
$$

Under $T$ symmetry Eq. (79) is a consequence of Eqs. (66 68). Eqs. (75)79) confirm that our expectation that the spin Hall current is robust against $T$ symmetry was indeed the case. A set of cancellation rules for the Hall type currents are established in Table 2.

\footnotetext{
${ }^{17}$ In the $I$-invariant case, instead of Eq. 65), Eq. 85 holds, i.e., the $I$-invariance of $H_{0}$ relates $\left\langle-\mathbf{x}, a \mid u_{i}(-\mathbf{k})\right\rangle$ with $\left\langle\mathbf{x}, \tau \mid u_{j}(\mathbf{k})\right\rangle$ up to a $S U(N)$ gauge degree of freedom $h$. This reduces in terms of spin and field strength to

$$
\begin{aligned}
\left\langle S_{\alpha}\right\rangle(-\mathbf{k}) & =h^{-1}\left\langle S_{\alpha}\right\rangle(\mathbf{k}) h, \\
\mathcal{F}_{k_{\mu} k_{\nu}}(-\mathbf{k}) & =h^{-1} \mathcal{F}_{k_{\mu} k_{\nu}}(\mathbf{k}) h .
\end{aligned}
$$

Eq. (77) justifies [76), whereas multiplying Eqs. (77) and [78), one finds immediately Eq. (79). 


\section{$5.3 \mathcal{F}_{k_{\mu} t}$ induces a polarization current: parity polariza- tion current and quantum spin pump}

We have seen in the previous section that $\mathcal{F}_{k_{\mu} k_{\nu}}$ is related to Hall type currents associated with the internal degrees of freedom such as charge and spin, by applying a uniform electric field to the system. Here we argue that $\mathcal{F}_{k_{\mu}} t$ describes various kinds of polarization current. More specifically, we consider a situation where a band insulator is subject to a time-dependent perturbation which does not break the periodicity of the underlying crystal, i.e., we consider a Hamiltonian,

$$
H(\mathbf{x}, \mathbf{p} ; \beta(t))=H(\mathbf{x}+\mathbf{a}, \mathbf{p} ; \beta(t)) .
$$

Since the perturbation $\beta(t)$ does not depend on $\mathbf{x}$, the local Hamiltonian defined in Eq. (12) reduces simply to

$$
H_{\mathrm{loc}}(\mathbf{x}, \mathbf{p}, t)=H(\mathbf{x}, \mathbf{p} ; \beta(t)) .
$$

Depending on the perturbation $\beta(t)$, the ground state wave function of the local Hamiltonian, $H_{\text {loc }}(\mathbf{x}, \mathbf{p}, t)$ also evolves temporally. Since an electronic wave function for the ground state describes spatial distributions of charge, spin and orbital, its evolution in general induces various kinds of currents in the system. When the system is isolated from the external circuit, an induced current accumulates an extra charge (or spin, orbital) on one side of the system, which results in a spatial polarization of charge, spin and orbital. 39 Accordingly, this type of current associated with such internal degrees of freedom is often called a polarization current. In the following, we describe the physics of polarization current using the language of non-abelian gauge field, in particular, that of $\mathcal{F}_{k_{\mu} t}$. One of the advantges of taking such a viewpoint is that the role of symmetry becomes transparent, which we summarized as a set of cancellation rules in Table 2.

\subsubsection{Charge polarization current}

Let us first consider a charge polarization current. In the case of timedependent perturbation (80), the EOM analogous to Eqs. (6162), are found to be

$$
\begin{aligned}
\frac{d \bar{x}_{\mu}^{(j)}}{d t} & =\frac{\partial \epsilon_{\mathrm{loc}}(\mathbf{k})}{\partial \bar{k}_{\mu}}-\mathbf{z}^{(j) \dagger} \mathcal{F}_{k_{\mu} t}(\overline{\mathbf{k}}, t) \mathbf{z}^{(j)}, \\
\frac{d \bar{k}_{\mu}^{(j)}}{d t} & =0
\end{aligned}
$$

where $j=1, \cdots, N$. Collecting contributions from all $N$ pseudospin states and from all filled $\overline{\mathbf{k}}$ points, one can calculate the charge current carried by the ground state as

$$
\left(\mathbf{J}_{\mathcal{C}}^{\mathrm{pol}}\right)_{\mu}=-e \int_{\mathrm{BZ}} \frac{d \overline{\mathbf{k}}}{(2 \pi)^{D}} \sum_{j=1}^{N} \frac{d \bar{x}_{\mu}^{(j)}}{d t}
$$




$$
=e \int_{\mathrm{BZ}} \frac{d \overline{\mathbf{k}}}{(2 \pi)^{D}} \operatorname{Tr}\left[\mathcal{F}_{k_{\mu} t}(\overline{\mathbf{k}}, t)\right]
$$

where the $\overline{\mathbf{k}}$-integral was performed over the whole Brillouin zone (BZ). Eq. (83) is analogous to Eq. (64), which we found for the charge Hall current. We can see that the trace of different types of reciprocal field strength, i.e., $\mathcal{F}_{k_{\mu} t}$ and $\mathcal{F}_{k_{\mu} k_{\nu}}$ are related to different types of physical currents, i.e., polarization and Hall type currents.

We have seen in the previous section that no charge Hall current flows whenever the sysytem is invariant under time reversal $T$. In contrast, we are going to see below that the charge polarization current vanishes whenever the system is invariant under spatial inversion $I,{ }^{18}$

$$
H_{\mathrm{loc}}(\mathbf{x}, \mathbf{p}, t)=H_{\mathrm{loc}}(-\mathbf{x},-\mathbf{p}, t) .
$$

In this case, its $N$-fold degenerate Bloch functions at $\mathbf{k}$ is related to those at - k upto a certain $S U(N)$ gauge transformation $h(\mathbf{k}, t)$,

$$
\left\langle-\mathbf{x}, a \mid u_{i}(-\mathbf{k}, t)\right\rangle=\sum_{j=1}^{N}\left\langle\mathbf{x}, a \mid u_{j}(\mathbf{k}, t)\right\rangle h_{j i}(\mathbf{k}, t) .
$$

Since the field strength $\mathcal{F}_{k_{\mu} t}$ is related through Eqs. (78) to those wavefunctions, Eq. (85) reduces to the following identity,

$$
\mathcal{F}_{k_{\mu} t}(-\mathbf{k}, t)=-h^{-1} \mathcal{F}_{k_{\mu} t}(\mathbf{k}, t) h
$$

Consequently,

$$
\operatorname{Tr}\left[\mathcal{F}_{k_{\mu} t}(-\mathbf{k}, t)\right]=-\operatorname{Tr}\left[\mathcal{F}_{k_{\mu} t}(\mathbf{k}, t)\right] .
$$

Eqs. [83) and (87) indicate that the charge polarization current always vanishes in $I$-invariant systems.

\subsubsection{Parity polarization current}

We have already encountered a similar situation in the previous section. Under the time reversal $T, \mathcal{F}_{k_{\mu} k_{\nu}}(\mathbf{k})$ is transformed to $-\left(\mathcal{F}_{k_{\mu} k_{\nu}}(-\mathbf{k})\right)^{t}$ upto a $S U(N)$ gauge degree of freedom, as can seen in Eq. (66). As a result, the charge Hall current vanished in $T$-invariant systems. On the other hand, a spin Hall current was robust against $T$-invariance. The reason was that not only $\mathcal{F}_{k_{\mu} k_{\nu}}$ but also the spin operator are odd under the time-reversal, as given respectively in Eqs. (66) and (68).

Following the same type of logic, we can expect that an orbital polarization current may remain finite irrespective of the $I$-invariance of $H_{\text {loc }}$, as far as the associated orbital operator $\Pi(\mathbf{x}, \mathbf{p})$ changes its sign under the spatial inversion

\footnotetext{
18 i.e., the underlying crystal structure has centro-symmetric points.
} 
I. 19 Accordingly we dub this type of orbital current a parity polarization current.

Expecting that the above analogy is indeed a sensible one, let us further analyze the parity polarization current carried by the ground state. Since we have defined this orbital current as Eq. (5253), we have to consider an EOM for

$$
\mathcal{O}_{\mu}^{\Pi}=\mathcal{O}_{\mu}(\Pi(\mathbf{x}, \mathbf{p}))=\frac{1}{2}\left(\Pi x_{\mu}+x_{\mu} \Pi\right) .
$$

We derive their EOM in terms of the decomposition (33). Our local Hamiltonian is time-dependent, and so is its local Bloch function. Therefore, $\Omega_{\mathcal{O}_{\mu}^{\Pi}}^{(2)}$ appearing in Eq. (33) remains finite in general:

$$
\begin{aligned}
& \frac{d \overline{\mathcal{O}}_{\mu}^{\Pi}}{d t}=\Omega_{\mathcal{O}_{\mu}^{\Pi}}^{(1)}+\Omega_{\mathcal{O}_{\mu}^{\Pi}}^{(2)} \\
= & i \sum_{m, n=1}^{N} \int d \mathbf{k} d \mathbf{k}^{\prime} a_{m}^{*}(\mathbf{k}, t)\left[\left\langle H_{\mathrm{loc}}\right\rangle-i \nabla_{t},\left\langle\mathcal{O}_{\mu}^{\Pi}\right\rangle\right]_{m n}\left(\mathbf{k}, \mathbf{k}^{\prime}\right) a_{n}\left(\mathbf{k}^{\prime}, t\right) .
\end{aligned}
$$

This equation is analogous to Eq. (72). The covariant derivative $\nabla_{k_{\mu}}=\left\langle x_{\mu}\right\rangle$ in Eq. (72) was replaced in Eq. (89) by another covariant derivative w.r.t. time, i.e., $\nabla_{t}$ :

$$
\left(i \nabla_{t}\right)_{m n}=\frac{\partial}{\partial t} \delta_{m n}-i\left(\mathcal{A}_{t}\right)_{m n} .
$$

Let us further develop the analogy between the two cases, i.e., we rewrite Eq. (89) in the following way, precisely as we rewrote (72) as (73). The details of the derivation is given in Appendix D, which is in parallel with Appendix C, and the result is

$$
\begin{aligned}
\frac{d \overline{\mathcal{O}}_{\mu}^{\Pi}}{d t} & =\sum_{m, n=1}^{N} \int d \mathbf{k} \rho(\mathbf{k}) z_{m}^{*}(\mathbf{k}, t)\langle\Pi\rangle_{m n}(\mathbf{k}) \frac{\partial \epsilon_{\mathrm{loc}}(\mathbf{k})}{\partial k_{\mu}} z_{n}(\mathbf{k}, t) \\
& -\frac{1}{2} z_{m}^{*}(\mathbf{k}, t)\left(\langle\Pi\rangle(\mathbf{k}) \mathcal{F}_{k_{\mu}}(\mathbf{k})+\text { h.c. }\right)_{m n} z_{n}(\mathbf{k}, t) .
\end{aligned}
$$

Following the prescription (18), we see that the parity polarization current carried by $\mathbf{k}$ Bloch electron occupying the $j$-th pseudospin state is given by,

$$
\begin{aligned}
\frac{d \mathcal{O}_{\mu}^{\Pi(j)}}{d t} & =\mathbf{z}^{(j) \dagger}\langle\Pi\rangle(\overline{\mathbf{k}}) \mathbf{z}^{(j)} \frac{\partial \epsilon_{\text {loc }}(\overline{\mathbf{k}})}{\partial \bar{k}_{\mu}} \\
& -\left(\mathbf{z}^{(j) \dagger}\langle\Pi\rangle(\overline{\mathbf{k}}) \mathcal{F}_{k_{\mu} t}(\overline{\mathbf{k}}) \mathbf{z}^{(j)}+\text { c.c. }\right) .
\end{aligned}
$$

After taking its summation over $N$ pseudo-spin states and over filled $\overline{\mathbf{k}}$ points, we finally obtain a parity polarization current carried by the ground state,

$$
\left(\mathbf{J}_{\mathcal{O}}^{\mathrm{pol}}\right)_{\mu}=\int_{\mathrm{BZ}} \frac{d \overline{\mathbf{k}}}{(2 \pi)^{D}} \sum_{j=1}^{N} \frac{d \overline{\mathcal{O}}_{\mu}^{\Pi(j)}}{d t}
$$

\footnotetext{
${ }^{19}$ See Eq. (56).
} 


$$
=-\int_{\mathrm{BZ}} \frac{d \overline{\mathbf{k}}}{(2 \pi)^{D}} \operatorname{Tr}\left[\langle\Pi\rangle(\overline{\mathbf{k}}) \mathcal{F}_{k_{\mu} t}(\overline{\mathbf{k}})\right] .
$$

The first term of Eq. (92) did not contribute to Eq. (93) due to a cancellation associated with the $T$-invariance, ${ }^{20}$

$$
\int_{\mathrm{BZ}} \frac{d \overline{\mathbf{k}}}{(2 \pi)^{D}} \operatorname{Tr}[\langle\Pi\rangle(\overline{\mathbf{k}})] \frac{\partial \epsilon_{\mathrm{loc}}(\overline{\mathbf{k}})}{\partial \bar{k}_{\mu}}=0 .
$$

On the contrary, the Berry phase contribution, i.e., Eq. (93) turns to be quite robust against both $I$ and $T$ symmetries. In particular, in the $I$-invariant case, $\langle\Pi\rangle(\mathbf{k})$ is identical to $-\langle\Pi\rangle(-\mathbf{k})$ up to the $S U(N)$ gauge transformation $h$,

$$
\langle\Pi\rangle(-\mathbf{k})=-h^{-1}\langle\Pi\rangle(\mathbf{k}) h
$$

This can be shown explicitly using Eqs. (56578554). Eqs. (8697) indicate

$$
\operatorname{Tr}\left[\langle\Pi\rangle(\mathbf{k}) \mathcal{F}_{k_{\mu} t}(\mathbf{k})\right]=\operatorname{Tr}\left[\langle\Pi\rangle(-\mathbf{k}) \mathcal{F}_{k_{\mu} t}(-\mathbf{k})\right] .
$$

Eq. (98) holds also true in the $T$ invariant case, as is clear from Eqs. 9495). Eqs. (9398) confirm our hypotheses that the parity polarization current is indeed robust against $I$ symmetry.

\subsubsection{Quantum spin pump}

Another possible direction to be explored is to study how to induce a spin polarization current by breaking both $T$-invariance and $I$-invariance. This scenario can be implemented [15] in a certain kind of quantum spin chains such as $\mathrm{Cu}$-bensoate and $\mathrm{Yb}_{4} \mathrm{As}_{3}$. The ground state of these quantum magnets is known to be quantum critical point (QCP), which is interpreted as a Dirac monopole, i.e., a source of the $\mathrm{U}(1)$ field strength $\mathcal{F}_{k t}$. When this quantum system is driven around this QCP by applying an electric field $\mathbf{E}$ and/or magnetic field $\mathbf{B}$, a spin polarization current can be induced. The electromagnetic fields break both the $I$-invariance and $T$-invariance. They also induce a spin gap and realize a quantum critical point at the origin of $\mathbf{E}-\mathbf{B}$ plane. When the system goes adiabatically around this origin, a quantized number of spins will be transported from one edge to the other through the system. This quantized value is a physical manifestation of the first Chern number associated with the QCP.

${ }^{20} T$-invariance relates $N$-fold degenerate Bloch functions at $\mathbf{k}$ with the ones at $-\mathbf{k}$ upto a $S U(N)$ gauge degree of freedom $g$ as Eq. 65. This implies,

$$
\begin{aligned}
(\langle\Pi\rangle(-\mathbf{k}))^{t} & =g^{-1}\langle\Pi\rangle(\mathbf{k}) g, \\
\left(\mathcal{F}_{k_{\mu} t}(-\mathbf{k})\right)^{t} & =g^{-1} \mathcal{F}_{k_{\mu} t}(\mathbf{k}) g .
\end{aligned}
$$

Eq. 94 justifies (96), whereas multiplying Eq. (94) with Eq. 95 one finds immediately (98). 


\section{4 $\mathcal{F}_{k_{\mu} x_{\mu}}$ associated with the spatial inhomogeneity}

Contrary to $\mathcal{F}_{k_{\mu} k_{\nu}}$ and $\mathcal{F}_{k_{\mu} t}$, the reciprocal field strength $\mathcal{F}_{k_{\mu} x_{\nu}}$ does not seem to be related directly to a physical observable such as Hall type currents and polarization currents. However, when the system contains spatial inhomogeneity such as lattice defects, $\mathcal{F}_{k_{\mu} x_{\nu}}$ appears and plays an important role in the dynamics of Bloch electrons around the defects [7]. Another possible application of $\mathcal{F}_{k_{\mu} x_{\nu}}$ is the electron transport properties around a magnetic domain wall, where the spatial modulation of ferromagnetic moments induce $\mathcal{F}_{k_{\mu} x_{\nu}}$, and naturally influences the EOM for the electron wave packet through this Berry curvature.

\section{Conclusions}

We have derived and analyzed the semiclassical EOM for a wave packet of Bloch electrons, under perturbations slowly varying in space and in time. Their interpretation in terms of non-abelian gauge field in the reciprocal parameter space was the central issue of the paper. The same type of EOM has been previously derived for the abelian, i.e., U(1) case, by using either (i) Timedependent variational principle [7] or (ii) Path-integral method using Wannier basis [30. We have generalized such EOM to a non-abelian case by using only the most fundamental principles of quantum mechanics.

The advantage of our formalism was that

1. it was asymptotically exact in the framework of linear response theory, as a result of systematic expansion w.r.t. the perturbation $\beta$ or $\mathbf{x}-\overline{\mathbf{x}}$,

2. it revelaed that there are different types of gauge field of different physical origin,

3. it was useful for developing symmetry analyses on various types of Berry phase transport.

The first point refers to Eq. (20) and all the related analyses developped in Secs. 3 and 4 . The relevance of our results in relation to other approaches was further discussed in Sec. 4.4. As for the second point, two different sources of gauge field have been revealed, i.e., (i) Projection onto a subspace spanned by $N$ Bloch bands, (ii) Bloch basis moving in the course of time. The former is the origin of $\mathcal{F}_{k_{\mu} k_{\nu}}$ which is directly related to spontaneous Hall currents of various degrees of freedom. The latter brings about $\mathcal{F}_{k_{\mu} x_{\mu}}$ and $\mathcal{F}_{k_{\mu} t}$, which plays an important role in the spatially and temporally inhomogeneous system.

Finally, concerning the last point in the above list, we have applied our formalism to the analyses on the spin and orbital transport phenomena with the help of Boltzmann transport theory. The role of time reversal and space inversion symmetries in the appearence of finite Hall/polarization currents has been extensively studied. The cancellation rules are summarized in Tables 1 and 
2. The concept of parity polarization current has also been introduced, which may concretize Berry phase engineering in the context of orbital transport.

We leave for a future study further investigations on their application to the domain wall physics and that of quantum pumping. In conclusion, we believe that our analyses on non-Abelian gauge field will see in the near future a possible application in the context of Berry phase engineering.

Note added - After completion of this work, we were informed of a related effort by D. Culcer, Y. Yao and Q. Niu. 40]

\section{Acknowledgements}

We would like to thank Shuichi Murakami and Naoto Nagaosa for introducing us into this flourishing area of physics, as well as giving us a motivation to work on this problem. We are also grateful to Dimitrie Culcer and Qian Niu for their comments and suggestions on our paper.

R.S. is a JSPS Postdoctoral Fellow. K.I. is supported by RIKEN as a Special Postdoctoral Researcher.

\section{Appendix A : Matrix element $\langle H\rangle_{m n}(\mathbf{k})$ and $\Delta \Omega_{x_{\mu}}^{(1)}$}

Matrix elements of the linearlized Hamiltonian, i.e., Eqs. (36 37), have been extensively used in Sec. 4.3. Let us recall those equations together with the matrix elements of noncommutative coordinates, i.e., Eqs. (23]27/25/28). Our purpose here is to substitute the expression (37) into

$$
\Omega_{x_{\mu}}^{(1)}=i \sum_{m, n=1}^{N} \int d \mathbf{k} a_{m}^{*}(\mathbf{k})\left[\langle H\rangle,\left\langle x_{\mu}\right\rangle\right]_{m n}(\mathbf{k}) a_{n}(\mathbf{k}),
$$

an expression analogous to the second line of Eqs. (33), and to rewrite $\Omega_{x_{\mu}}^{(1)}$ in terms of the field strength $\left[\mathcal{F}_{k_{\mu}} k_{\nu}\right]_{m n}$.

The first term of Eq. (37), i.e., $\epsilon_{\mathrm{loc}}(\mathbf{k}, \overline{\mathbf{x}}, t)$ gives a standard velocity term when inserted into Eq. (99). Since $\left[\mathcal{F}_{k_{\mu} k_{\nu}}\right]_{m n}$ is related to the commutator, $\left[\nabla_{k_{\mu}}, \nabla_{k_{\nu}}\right]_{m n}$ or equivalently, $\left[\left\langle x_{\mu}\right\rangle,\left\langle x_{\nu}\right\rangle\right]_{m n}(\mathbf{k})$, one can easily imagine that the last two terms give in Eq. (37) give when inserted into Eq. (99) a contribution related to $\left[\mathcal{F}_{k_{\mu} k_{\nu}}\right]_{m n}$. One can indeed verify

$$
\begin{aligned}
& -\left[\frac{1}{2}\left(\frac{\partial \epsilon_{\mathrm{loc}}}{\partial \bar{x}_{\nu}} \nabla_{k_{\nu}}+\nabla_{k_{\nu}} \frac{\partial \epsilon_{\mathrm{loc}}}{\partial \bar{x}_{\nu}}\right), i \nabla_{k_{\mu}}\right]_{m n} \\
= & -\frac{i}{2}\left\{\frac{\partial \epsilon_{\mathrm{loc}}}{\partial \bar{x}_{\nu}}\left[\nabla_{k_{\nu}}, \nabla_{k_{\mu}}\right]_{m n}+\sum_{l=1}^{N}\left[\frac{\partial \epsilon_{\mathrm{loc}}}{\partial \bar{x}_{\nu}}, \nabla_{k_{\mu}}\right]_{m l}\left[\nabla_{k_{\nu}}\right]_{l n}\right. \\
+ & \left.\sum_{l=1}^{N}\left[\nabla_{k_{\nu}}\right]_{m l}\left[\frac{\partial \epsilon_{\mathrm{loc}}}{\partial \bar{x}_{\nu}}, \nabla_{k_{\mu}}\right]_{l n}+\left[\nabla_{k_{\nu}}, \nabla_{k_{\mu}}\right]_{m n} \frac{\partial \epsilon_{\mathrm{loc}}}{\partial \bar{x}_{\nu}}\right\} \\
= & -\frac{\partial \epsilon_{\mathrm{loc}}}{\partial \bar{x}_{\nu}} \mathcal{F}_{k_{\nu} k_{\mu}}+\frac{i}{2}\left(\frac{\partial^{2} \epsilon_{\mathrm{loc}}}{\partial k_{\mu} \partial \bar{x}_{\nu}}\left[\nabla_{k_{\nu}}\right]_{m n}+\left[\nabla_{k_{\nu}}\right]_{m n} \frac{\partial^{2} \epsilon_{\mathrm{loc}}}{\partial k_{\mu} \partial \bar{x}_{\nu}}\right) .
\end{aligned}
$$


The second term of Eq. (37) gives, when inserted into the commutator,

$$
\left[\frac{\partial \epsilon_{\mathrm{loc}}(\mathbf{k}, \overline{\mathbf{x}}, t)}{\partial \bar{x}_{\nu}} \bar{x}_{\nu}, \nabla_{k_{\mu}}\right]_{m n}=-\frac{\partial^{2} \epsilon_{\mathrm{loc}}}{\partial k_{\mu} \partial \bar{x}_{\nu}} \bar{x}_{\nu}
$$

Collecting the contribution (101) and the last two terms of Eq. (100), i.e., terms not related to $\mathcal{F}_{k_{\nu} k_{\mu}}$, one defines $\Delta \Omega_{x_{\mu}}^{(1)}$ introduced in Eq. (38) ;

$$
\Delta \Omega_{x_{\mu}}^{(1)}=-\sum_{\nu, m, n} \int d \mathbf{k} \frac{\partial^{2} \epsilon_{\mathrm{loc}}}{\partial k_{\mu} \partial \bar{x}_{\nu}} \rho(\mathbf{k}, t)\left\{\bar{x}_{\nu}(t)-i z_{m}^{*}(\mathbf{k}, t)\left[\nabla_{k_{\nu}}\right] z_{n}(\mathbf{k}, t)\right\}
$$

This term vanishes after $k$-integration with the help of prescription given in Eq. (18).

\section{Appendix B : Derivation of the EOM for $\overline{\mathbf{z}}(t)$}

We demonstrate here the derivation of EOM for $\overline{\mathbf{z}}(t)$, i.e., EOM describing the motion of the internal pseudospin degree of freedom. For that purpose we once have to go back to Eq. (30). After multiplying it with a weight $\sqrt{\rho(\mathbf{k}, t)}$, we integrate it over all the $\mathbf{k}$-points, to find,

$$
\begin{aligned}
& \int d \mathbf{k}\left\{\frac{1}{2} \frac{\partial}{\partial t}(\rho(\mathbf{k}, t)) \mathbf{z}(\mathbf{k}, t)+\rho \frac{\partial \mathbf{z}}{\partial t}\right\} \\
= & i \int d \mathbf{k} \rho(\mathbf{k}, t)\left\{-\epsilon_{\mathrm{eff}}+\bar{x}_{\mu} \frac{\partial \epsilon_{\mathrm{loc}}}{\partial \bar{x}_{\mu}}-\frac{\partial \epsilon_{\mathrm{loc}}}{\partial \bar{x}_{\mu}} A_{k_{\mu}}+\frac{d \bar{x}_{\mu}}{d t} \mathcal{A}_{\bar{x}_{\mu}}+\mathcal{A}_{t}\right\} \mathbf{z} \\
+ & \frac{1}{2} \int d \mathbf{k} \rho(\mathbf{k}, t) \frac{\partial \epsilon_{\mathrm{loc}}}{\partial \bar{x}_{\mu}} \frac{\partial \mathbf{z}}{\partial k_{\mu}}
\end{aligned}
$$

where $\epsilon_{\text {eff }}=\epsilon_{\mathrm{loc}} 1+\Delta \epsilon$ as given in Eq. (38). In Eq. (103), repeated $\mu$-indices should be summed over $\mu=1, \cdots, D$. In order to obtain Eq. (103), we also used,

$$
\int d \mathbf{k}\left\{\sqrt{\rho} \frac{\partial \epsilon_{\text {loc }}(\overline{\mathbf{k}})}{\partial \bar{x}_{\mu}} \frac{\partial}{\partial k_{\mu}}(\sqrt{\rho} \mathbf{z})+\frac{1}{2} \rho \mathbf{z} \frac{\partial \epsilon_{\text {loc }}}{\partial k_{\mu} \partial \bar{x}_{\mu}}\right\}=\frac{1}{2} \int d \mathbf{k} \rho \frac{\partial \epsilon_{\text {loc }}}{\partial \bar{x}_{\mu}} \frac{\partial \mathbf{z}}{\partial k_{\mu}} .
$$

We now substitute,

$$
\frac{\partial}{\partial t}(\rho(\mathbf{k}, t))=\sum_{\mu=1}^{D} \frac{\partial}{\partial k_{\mu}}\left\{\rho(\mathbf{k}, t) \frac{\partial \epsilon_{\mathrm{loc}}(\mathbf{k}, \overline{\mathbf{x}}, t)}{\partial \bar{x}_{\mu}}\right\},
$$

into Eq. (103), then perform a partial integral w.r.t. $k_{\mu}$. The result is,

$$
\begin{aligned}
& \int d \mathbf{k} \rho(\mathbf{k}, t)\left\{-\frac{\partial \epsilon_{\mathrm{loc}}}{\partial \bar{x}_{\mu}} \frac{\partial \mathbf{z}}{\partial k_{\mu}}+\frac{\partial \mathbf{z}}{\partial t}\right\} \\
& =i \int d \mathbf{k} \rho\left\{-\epsilon_{\mathrm{eff}}+\bar{x}_{\mu} \frac{\partial \epsilon_{\mathrm{loc}}}{\partial \bar{x}_{\mu}}-\frac{\partial \epsilon_{\mathrm{loc}}}{\partial \bar{x}_{\mu}} A_{k_{\mu}}+\frac{d \bar{x}_{\mu}}{d t} \mathcal{A}_{\bar{x}_{\mu}}+\mathcal{A}_{t}\right\} \mathbf{z}
\end{aligned}
$$


Finally, in order to rewrite Eq. (105) in the form of Eq. (44) and complete its derivation, we adopt the prescritpion (18).

\section{Appendix C : EOM for $\mathcal{O}_{\mu \alpha}^{\mathcal{S}}$ and spin Hall current}

Our purpose here is to rewrite an EOM for Eq. (72) into its final form, i.e., Eq. (73), so that we can express the spin Hall current as the following trace in the pseudospin space, $\operatorname{Tr}\left[\left\langle S_{\alpha}\right\rangle(\overline{\mathbf{k}}) \mathcal{F}_{\bar{k}_{\mu} \bar{k}_{\nu}}(\overline{\mathbf{k}})\right] E_{\nu}$.

Let us first recall the assumption we made in Eq. (58). This assumption allows us to factorize $\left\langle\mathcal{O}_{\mu \alpha}^{\mathcal{S}}\right\rangle_{m n}\left(\mathbf{k}, \mathbf{k}^{\prime}\right)$ in Eq. (72) into a product of $\left\langle S_{\alpha}\right\rangle$ and $\left\langle x_{\mu}\right\rangle$;

$$
\left\langle S_{\alpha} x_{\mu}+x_{\mu} S_{\alpha}\right\rangle_{m n}=\sum_{l=1}^{N}\left(\left\langle S_{\alpha}\right\rangle_{m l}\left\langle x_{\mu}\right\rangle_{l n}+\left\langle x_{\mu}\right\rangle_{m l}\left\langle S_{\alpha}\right\rangle_{l n}\right) .
$$

Correspondingly, the commutator appearing in Eq. (72) can be decoupled into the following two types of commutators,

$$
\left[\langle H\rangle,\left\langle\mathcal{O}_{\mu \alpha}^{\mathcal{S}}\right\rangle\right]=\frac{1}{2}\left[\langle H\rangle,\left\langle S_{\alpha}\right\rangle\right]\left\langle x_{\mu}\right\rangle+\frac{1}{2}\left\langle S_{\alpha}\right\rangle\left[\langle H\rangle,\left\langle x_{\mu}\right\rangle\right]-\text { h.c. }
$$

The first term together with its hermitian conjugate conceives a commutator between $\langle H\rangle$ and $\left\langle S_{\alpha}\right\rangle$, which consitutes the EOM for the spin : $\frac{d S_{\alpha}}{d t}$. Firstly, we show that this commutator vanishes. Since $\left\langle S_{\alpha}\right\rangle$ is diagonal in $\mathbf{k}$-space, it clearly commutes with $\left\langle H_{0}\right\rangle$;

$$
\begin{aligned}
{\left[\left\langle H_{0}\right\rangle,\left\langle S_{\alpha}\right\rangle\right]_{m n}\left(\mathbf{k}^{\prime}, \mathbf{k}\right) } & =\delta\left(\mathbf{k}^{\prime}-\mathbf{k}\right)\left[\epsilon_{\mathrm{loc}}(\mathbf{k}),\left\langle S_{\alpha}\right\rangle(\mathbf{k})\right]_{m n} \\
& =0
\end{aligned}
$$

Therefore, the commutator between $\langle H\rangle$ and $\left\langle S_{\alpha}\right\rangle$ becomes proportional to the covariant derivative of $\left\langle S_{\alpha}\right\rangle$ (k) w.r.t. $k_{\mu}$, i.e.,

$$
\begin{aligned}
& {\left[\langle H\rangle,\left\langle S_{\alpha}\right\rangle\right]_{m n}\left(\mathbf{k}^{\prime}, \mathbf{k}\right)=e \sum_{\mu=1}^{D} E_{\mu}\left[\left\langle x_{\mu}\right\rangle,\left\langle S_{\alpha}\right\rangle\right]_{m n}\left(\mathbf{k}^{\prime}, \mathbf{k}\right)} \\
& =i e \delta\left(\mathbf{k}^{\prime}-\mathbf{k}\right) \sum_{\mu=1}^{D} E_{\mu}\left[\nabla_{k_{\mu}},\left\langle S_{\alpha}\right\rangle(\mathbf{k})\right]_{m n} .
\end{aligned}
$$

Because the spin operator itself does not depend on the crystal momentum, the partial derivative of $\left\langle S_{\alpha}\right\rangle$ (k) w.r.t. $k_{\mu}$ reduces to the commutator between $i \mathcal{A}_{k_{\mu}}$ and $\left\langle S_{\alpha}\right\rangle(\mathbf{k})$, :

$$
\begin{aligned}
& \frac{\partial}{\partial k_{\mu}}\left\langle S_{\alpha}\right\rangle_{m n}(\mathbf{k})=\left\langle\frac{\partial u_{m}(\mathbf{k})}{\partial k_{\mu}}\left|S_{\alpha}\right| u_{n}(\mathbf{k})\right\rangle+\left\langle u_{m}(\mathbf{k})\left|S_{\alpha}\right| \frac{\partial u_{n}(\mathbf{k})}{\partial k_{\mu}}\right\rangle \\
& =\sum_{l=1}^{N}\left\{\left\langle\frac{\partial u_{m}}{\partial k_{\mu}} \mid u_{l}\right\rangle\left\langle u_{l}\left|S_{\alpha}\right| u_{n}\right\rangle+\left\langle u_{m}\left|S_{\alpha}\right| u_{l}\right\rangle\left\langle u_{l} \mid \frac{\partial u_{n}}{\partial k_{\mu}}\right\rangle\right\}
\end{aligned}
$$


In the second line $k$-dependence is not written explicitly. We used Eq. (58) between the two lines. Then the covariant derivative of $\left\langle S_{\alpha}\right\rangle(\mathbf{k})$ w.r.t. $k_{\mu}$ appearing in Eq. (108) also vanishes,

$$
\begin{aligned}
{\left[\nabla_{k_{\mu}},\left\langle S_{\alpha}\right\rangle(\mathbf{k})\right]_{m n} } & =\frac{\partial}{\partial k_{\mu}}\left(\left\langle S_{\alpha}\right\rangle_{m n}(\mathbf{k})\right)-\left[i \mathcal{A}_{k_{\mu}},\left\langle S_{\alpha}\right\rangle(\mathbf{k})\right]_{m n} \\
& =0
\end{aligned}
$$

Consequently, the first term and its hermitian conjugate in Eq. (106) are indeed zero. On the other hand, the second term in Eq. (106) contains the commutator between $\langle H\rangle$ and $\left\langle x_{\nu}\right\rangle$, which describes the EOM for $x_{\nu}$ now. This term gives rise to a field strength $\mathcal{F}_{k_{\mu} k_{\nu}}$ through the commutator between covariant derivatives w.r.t. different components of the crystal momentum, i.e., $\left[\nabla_{k_{\mu}}, \nabla_{k_{\nu}}\right]$. Namely,

$$
\begin{aligned}
{\left[\langle H\rangle,\left\langle x_{\nu}\right\rangle\right] } & =\left[\left\langle H_{0}\right\rangle+e \sum_{\mu=1}^{D} E_{\mu}\left\langle x_{\mu}\right\rangle,\left\langle x_{\nu}\right\rangle\right]\left(\mathbf{k}^{\prime}, \mathbf{k}\right) \\
& =\delta\left(\mathbf{k}^{\prime}-\mathbf{k}\right)\left[\epsilon_{\mathrm{loc}}(\mathbf{k}) \mathbf{1}+e \sum_{\mu=1}^{D} E_{\mu} i \nabla_{k_{\mu}}, i \nabla_{k_{\nu}}\right] \\
& =-i \delta\left(\mathbf{k}^{\prime}-\mathbf{k}\right)\left\{\frac{\partial \epsilon_{\mathrm{loc}}}{\partial k_{\nu}} \mathbf{1}-e \sum_{\mu=1}^{D} E_{\mu}\left(\mathcal{F}_{k_{\mu} k_{\nu}}(\mathbf{k})\right)\right\}
\end{aligned}
$$

Finally, substituting Eq. (106) together with Eqs. (108110) and (111) into Eq. (72), one finds Eq. (73).

\section{Appendix D : EOM for $\mathcal{O}_{\mu}^{\Pi}$ and parity polarization current}

In parallel with Appendix C, we rewrite below Eq. (89) into its final form, i.e., Eq. (91). Let first recall the assumption (59), which says that the parity operator $\Pi$ has no matrix element outside the $N$-fold degenerate band. This implies that $\left\langle\mathcal{O}_{\mu}^{\Pi}\right\rangle$ can be factorized into a product of two $N$ by $N$ matrices, or $\left\langle\Pi x_{\mu}\right\rangle=\langle\Pi\rangle\left\langle x_{\mu}\right\rangle$. Thanks to this factorization, the commutator in Eq. (89) can be decomposed into two types of commutators as

$$
\begin{aligned}
{\left[\left\langle H_{\mathrm{loc}}\right\rangle-i \nabla_{t},\left\langle\mathcal{O}_{\mu}^{\Pi}\right\rangle\right] } & =\frac{1}{2}\left(\left[\left\langle H_{\mathrm{loc}}\right\rangle-i \nabla_{t},\langle\Pi\rangle\right]\left\langle x_{\mu}\right\rangle+\text { h.c. }\right) \\
& +\frac{1}{2}\left(\langle\Pi\rangle\left[\left\langle H_{\mathrm{loc}}\right\rangle-i \nabla_{t},\left\langle x_{\mu}\right\rangle\right]+\text { h.c. }\right)
\end{aligned}
$$

On the r.h.s., the first term is a commutator between $\left\langle H_{\text {loc }}\right\rangle-i \nabla_{t}$ and $\langle\Pi\rangle$, which constitutes the EOM of the parity under time-dependent perturbations: $d \Pi / d t$. Since the parity operator itself is independent of time, we can prove that this commutator indeed vanishes, in the same way as we did in Eq. (109),

$$
\left[\left\langle H_{\mathrm{loc}}\right\rangle-i \nabla_{t},\langle\Pi\rangle\right]=0 \text {. }
$$

On the other hand, the second line of Eq. (112) is a commutator between $\left\langle H_{0}\right\rangle-i \nabla_{t}$ and $\left\langle x_{\mu}\right\rangle$, which describes the EOM for $x_{\mu}$. This commutator gives 
rise to $\mathcal{F}_{k_{\nu} t}$ through a commutation relation between two covariant derivatives,

one w.r.t. time and the other w.r.t. the momentum, $\left[i \nabla_{k_{\nu}}, i \nabla_{t}\right]=i \mathcal{F}_{k_{\nu} t}$. Thus the second line of Eq. (112) may be rewritten as

$$
\begin{aligned}
& {\left[\left\langle H_{\mathrm{loc}}\right\rangle-i \nabla_{t},\left\langle x_{\mu}\right\rangle\right]_{m n}\left(\mathbf{k}^{\prime}, \mathbf{k}\right)=i \delta\left(\mathbf{k}^{\prime}-\mathbf{k}\right)\left[\epsilon_{\mathrm{loc}}(\mathbf{k})-i \nabla_{t}, i \nabla_{k_{\mu}}\right]_{m n} } \\
= & -i \delta\left(\mathbf{k}^{\prime}-\mathbf{k}\right)\left\{\frac{\partial \epsilon_{\mathrm{loc}}(\mathbf{k})}{\partial k_{\nu}} \delta_{m n}-\left(\mathcal{F}_{k_{\mu} t}\right)_{m n}\right\} .
\end{aligned}
$$

Substituting Eq. (112) together with Eqs. (113114) into Eq. (89), one finds Eq. (911).

\section{References}

[1] P.A.M. Dirac, Proc. R. Soc. London 133, 60 (1931).

[2] G. 't Hooft, Nucl. Phys. B 79, 276 (1974); A.M. Polyakov, JETP Lett. 20, 194 (1974).

[3] M. Onoda, N. Nagaosa, J. Phys. Soc. Jpn. 71, 19 (2002).

[4] Z. Fang, et al., Science 302, 92 (2003).

[5] See also, G.E. Volovik, JETP Lett. 46, 98 (1987), and references therein.

[6] M.C. Chang, Q. Niu, Phys. Rev. Lett. 75, 1348 (1995); ibid., Phys. Rev. B 53, 7010 (1996).

[7] G. Sundaram, Q. Niu, Phys. Rev. B 59, 14915 (1999).

[8] J.E. Hirsch, Phys. Rev. Lett. 83, 1834 (1999).

[9] S. Zhang, Phys. Rev. Lett. 85, 393 (2000).

[10] S. Murakami, N. Nagaosa, S.C. Zhang, Science 301, 1348 (2003); ibid., Phys. Rev. B 69, 235206 (2004).

[11] J. Sinova, et al., Phys. Rev. Lett. 92, 126603 (2004).

[12] D. Culcer, et al., Phys. Rev. Lett. 93, 046602 (2004).

[13] S. Datta, B. Das, Appl. Phys. Lett. 56, 665 (1990).

[14] D. J. Thouless, Phys. Rev. B 27, 6083 (1983); Q. Niu, Phys. Rev. Lett. 64, $1812(1990)$.

[15] R. Shindou, cond-mat/0312668

[16] M.V. Berry, Proc. R. Soc. London, A 392, 45 (1984). 
[17] C.A. Mead, Chem. Phys. 49, 23 (1980)

[18] R. Karplus, J.M. Luttinger, Phys. Rev. 95, 1154 (1954).

[19] J. Zak, Phys. Rev. B 15, 771 (1977); ibid., 16, 4154 (1977).

[20] E.N. Adams and E.I. Blout, Phys. and Chem. Solids 10, 286, (1959); E.I. Blout, Solid State Phys. 13, 305, (1962).

[21] D.J. Thouless, et al., Phys. Rev. Lett. 49, 405 (1982).

[22] R.D. King-Smith, D. Vanderbilt, Phys. Rev. B 47, 1651 (1993); R. Resta, Europhys. Lett. 22, 133 (1993). See also, R. Resta, Rev. Mod. Phys. 66, 899 (1994), and references therein.

[23] K. Ohgushi, S. Murakami, N. Nagaosa, Phys. Rev. B 62, 6065 (2000).

[24] R. Shindou, N. Nagaosa, Phys. Rev. Lett. 87, 116801 (2001).

[25] T. Jungwirth, Q. Niu, A.H. MacDonald, Phys. Rev. Lett. 88, 207208 (2002).

[26] Y. Yao et al. Phys. Rev. Lett. 92, 037204 (2004).

[27] F. Wilczek, A. Zee Phys. Rev. Lett. 52, 2111 (1984).

[28] J. Inoue, G.E.W. Bauer, L.W. Molenkamp, Phys. Rev. B 70, R041303 (2004).

[29] J. Schliemann, D. Loss, Phys. Rev. B 69, 165315 (2004).

[30] H. Koizumi, Y. Takada, Phys. Rev. B 65, 153104 (2002).

[31] A. Berard, H. Mohrbach, Phys. Rev. D 69, 127701 (2004).

[32] N. Seiberg, E. Witten, J. High Energy Phys. 9909, 32 (1999).

[33] A. Connes, M.R. Douglas, A. Schwarz, J. High Energy Phys. 9802, 3 (1998).

[34] N. Marzari, D. Vanderbilt, Phys. Rev. B 56, 12847 (1997).

[35] K. Ishikawa, T. Matsuyama, Z. Phys. C 33, 41 (1986); ibid., Nucl. Phys. B 280, 523 (1987).

[36] S.C. Zhang, T.H. Hansson, S. Kivelson, Phys. Rev. Lett. 62, 82 (1989). See also, S.C. Zhang, Int. J. Mod. Phys. 6, 25 (1992).

[37] G.E. Volovik, JETP 67, 1804 (1988); J. Goryo, K. Ishikawa, Phys. Lett A 260, 294 (1999); A. Furusaki, M. Matsumoto, M. Sigrist, Phys. Rev. B 64, 054514 (2001).

[38] G.E. Volovik, V.M. Yakovenko, J. Phys.: Condens. Matter 1, 5263 (1989); T. Senthil, J.B. Marston, M.P.A. Fisher, Phys. Rev. B 60, 4245 (1999).

[39] N. Sai, K.M. Rabe, D. Vanderbilt, Phys. Rev. B 66, 104108 (2002).

[40] D. Culcer, Y. Yao, Q. Niu, cond-mat/0411285 


\begin{tabular}{|c|c|c|}
\hline invariance & $T$ & $I$ \\
\hline \hline charge : $\mathbf{1}$ & + & + \\
\hline spin $:\langle S\rangle(\mathbf{k})$ & - & + \\
\hline parity $:\langle\Pi\rangle(\mathbf{k})$ & + & - \\
\hline \hline Hall-type current: $\mathcal{F}_{k_{\mu} k_{\nu}}(\mathbf{k})$ & - & + \\
\hline polarization current: $\mathcal{F}_{k_{\mu} t}(\mathbf{k})$ & + & - \\
\hline
\end{tabular}

Table 1: Transformation properties under time reversal $T$ and spatial inversion $I$ - A negative (positive) sign indicates whether a matrix element in question at $\mathbf{k}$ reverses its sign (or not) in comparison with that of $-\mathbf{k}$ when the system is invariant under a certain symmetry operation, such as $T$ or $I$. For example, the negative sign for $\langle S\rangle(\mathbf{k})$ in the $T$-invariant case means that the spin matrix $\left\langle S_{\alpha}\right\rangle(\mathbf{k})$ is identical to $-\left(\left\langle S_{\alpha}\right\rangle(-\mathbf{k})\right)^{t}$ upto a certain $S U(N)$ gauge transformation as given in Eq.(68).

\begin{tabular}{|c|c|c|c|c|}
\hline type of current & \multicolumn{2}{|c|}{ Hall-type } & \multicolumn{2}{c|}{ polarization } \\
\hline invariance & $T$ & $I$ & $T$ & $I$ \\
\hline \hline charge & - & + & + & - \\
\hline spin & + & + & - & - \\
\hline parity & - & - & + & + \\
\hline
\end{tabular}

Table 2: Cancellation rules for charge/spin/parity Hall-type/polarization currents - A negative (positive) sign indicates that contributions to the total charge/spin/parity (vertical axis) Hall-type/polarization (horizontal axis) current from $\mathbf{k}$ and $\mathbf{- k}$ electrons (do not) cancel each other when the system is invariant under either $T$ or $I$. This table can be deduced from Table 1 . For example, in the $T$-invariant case, a negative sign for the spin in Table 1 gives, together with another negative sign for the Hall-type current, a positive sign for the spin Hall current in Table 2, correspoinding, respectively, to Eqs. 6866) and (79). 